\title{
Affine partitions and affine Grassmannians
}

\author{
Sara C. Billey * \\ Department of Mathematics \\ University of Washington \\ Seattle, WA \\ billey@math. washington.edu
}

\author{
Stephen A. Mitchell ${ }^{\dagger}$ \\ Department of Mathematics \\ University of Washington \\ Seattle, WA \\ mitchell@math. washington.edu
}

Submitted: Mar 25, 2008; Accepted: Jun 24, 2009; Published: Jul 2, 2009

Mathematics Subject Classification: 05E15, $14 \mathrm{M} 15$

\begin{abstract}
We give a bijection between certain colored partitions and the elements in the quotient of an affine Weyl group modulo its Weyl group. By Bott's formula these colored partitions give rise to some partition identities. In certain types, these identities have previously appeared in the work of Bousquet-Melou-Eriksson, ErikssonEriksson and Reiner. In other types the identities appear to be new. For type $A_{n}$, the affine colored partitions form another family of combinatorial objects in bijection with $(n+1)$-core partitions and $n$-bounded partitions. Our main application is to characterize the rationally smooth Schubert varieties in the affine Grassmannians in terms of affine partitions and a generalization of Young's lattice which refines weak order and is a subposet of Bruhat order. Several of the proofs are computer assisted.
\end{abstract}

\section{Introduction}

Let $W$ be a finite irreducible Weyl group associated to a simple connected compact Lie group $G$, and let $\widetilde{W}$ be its associated affine Weyl group. In analogy with the Grassmannian manifolds in classical type $A$, the quotient $\widetilde{W} / W$ is the indexing set for the Schubert varieties in the affine Grassmannians $\mathcal{L}_{G}$. Let $\widetilde{W}^{S}$ be the minimal length coset representatives for $\widetilde{W} / W$. Much of the geometry and topology for the affine Grassmannians can be studied from the combinatorics of $\widetilde{W}^{S}$ and vice versa. For example, Bott [6] showed that the Poincare series for the cohomology ring for the affine Grassmannian is equal to

${ }^{*}$ S.B. was supported by UW Royalty Research Grant.

†S.M. was supported by the National Science Foundation. 
the length generating function for $\widetilde{W^{S}}$, and this series can be written in terms of the exponents $e_{1}, e_{2}, \ldots, e_{n}$ for $W$ as

$$
P_{\widetilde{W}^{S}}(t)=\frac{1}{\left(1-t^{e_{1}}\right)\left(1-t^{e_{2}}\right) \cdots\left(1-t^{e_{n}}\right)}
$$

Bott's formula suggests there is a natural bijection between elements in $\widetilde{W}^{S}$ and a subset of partitions that preserves length. The goal of this paper is to give such a bijection which has useful implications in terms of the geometry, topology and combinatorics of affine Grassmannians and Bruhat order. We use Bott's formula to prove one direction of this bijection. The family of partitions in the image of this map is not the most obvious one: partitions whose parts are all in the set of exponents. Instead, we map $\widetilde{W}^{S}$ to a family of colored partitions we call affine partitions using a canonical factorization into segments. The segments are determined in general by the minimal length coset representatives in a corresponding finite Weyl group, hence there are only a finite number of them. In the simplest cases, the segments are the elements in the $W$-orbit of the special generator $s_{0}$ in $W^{S}$ acting on the left. In other cases, the map works best if we use a smaller set of segments and their images under an automorphism of the Dynkin diagram.

Using our bijection between $\widetilde{W}^{S}$ and affine partitions, there are three natural partial orders on affine partitions. Bruhat order and the left weak order on $\widetilde{W}^{S}$ are inherited from $\widetilde{W}$. Thus, the affine partitions also inherit these two poset structures via the bijection. In addition we will introduce a generalization of Young's lattice on affine partitions which refines the weak order and is refined by the Bruhat order.

In type $A_{n}$, Misra and Miwa [23] showed that $(n+1)$-core partitions are in bijection with $\widetilde{W}^{S}$. Björner-Brenti [3], Eriksson-Eriksson [12] and Lapointe-Morse [19] have shown that the elements of $\widetilde{W}^{S}$ are in bijection not only with $(n+1)$-core partitions, but also with $k$-bounded partitions, and skew shapes with no long hooks. Each of these related ${ }^{1}$ partition bijections has useful properties in terms of the geometry of affine Grassmannians.

Affine partitions in type $A$ give a new perspective on these well studied families. From the point of view of affine partitions though, type $A$ is harder than the other types $B_{n}, C_{n}, D_{n}, E_{6,7,8}, G_{2}, F_{4}$ because the weak order on segments is the most complicated. Therefore, type $A$ is covered last though the reader interested only in type $A$ can skip past the other type specific sections.

The segments and reduced factorizations have been used before in a wide variety of other work $[8,9,10,12,17,18,21,27]$ in various types connecting affine partitions with lecture hall partitions, Pieri type rules for the homology and cohomology of affine Grassmannians, and hypergeometric identities. However, none of the previous work seems to address the complete set of affine Weyl groups as we do in this article. More details on previous work are given after the definitions in Section 3.

As an application of the theory of affine partitions and the generalized Young's lattice, we will give a characterization of rationally smooth Schubert varieties in the affine

\footnotetext{
${ }^{1}$ The Björner-Brenti partitions are related to the Lapointe-Morse partitions via conjugation of the corresponding core partition; a process called $k$-conjugation in [17]. The Lapointe-Morse partitions are the same as the Eriksson ${ }^{2}$ partitions but they are described differently.
} 
Grassmannians $\mathcal{L}_{G}$. The smooth and rationally smooth Schubert varieties in $\mathcal{L}_{G}$ for all simple connected Lie groups $G$ have recently been characterized by the following theorem which was proved using different techniques. The description given here for the rationally smooth Schubert varieties complements our original description and relates these elements to the affine partitions.

Theorem 1. [2] Let $X_{w}$ be the Schubert variety in $\mathcal{L}_{G}$ indexed by $w \in \widetilde{W}^{S}$.

1. $X_{w}$ is smooth if and only if $X_{w}$ is a closed parabolic orbit.

2. $X_{w}$ is rationally smooth if and only if one of the following conditions holds:

a) $X_{w}$ is a closed parabolic orbit.

b) The set of all $v \in \widetilde{W}^{S}$ such that $v \leq w$ in Bruhat order is totally ordered.

c) $W$ has type $A_{n}$ and $X_{w}$ is spiral (see Section 9 for definition).

d) $W$ has type $B_{3}$ and $w=s_{3} s_{2} s_{0} s_{3} s_{2} s_{1} s_{3} s_{2} s_{0}$ using the labeling of the Coxeter graph on Page 43.

By Theorem 1, we will say $w$ is a cpo if $X_{w}$ is a closed parabolic orbit if and only if $X_{w}$ is smooth. In terms of Bruhat order, the cpo's can be identified as follows. Let $\widetilde{S}$ be a generating set for $\widetilde{W}$ and let $I_{w}=\{s \in \widetilde{S}: s \leq w\}$. Then $w \in \widetilde{W}^{S}$ is a cpo if and only if $s w \leq w$ for all $s \in I_{w}$.

For our characterization of rational smoothness, we will rely on the following theorem due to Carrell and Peterson which requires a bit more terminology. It is known that the Poincaré polynomial of the Schubert variety $X_{w}$ in $\mathcal{L}_{G}$ is determined by

$$
P_{w}(t)=\sum t^{\ell(v)}
$$

where the sum is over all $v$ in $\widetilde{W}^{S}$ such that $v \leq w$ in Bruhat order on $\widetilde{W}$. See [16] for details. We say that a polynomial $F(t)=f_{0}+f_{1} t+f_{2} t^{2}+\cdots+f_{k} t^{k}$ is palindromic if $f_{i}=f_{k-i}$ for all $0 \leq i \leq k$.

Theorem 2. [11] Let $X_{w}$ be the Schubert variety in $\mathcal{L}_{G}$ indexed by $w \in \widetilde{W}^{S}$. Then $X_{w}$ is rationally smooth if and only if $P_{w}(t)$ is palindromic.

In light of Theorem 2, we will say $w \in \widetilde{W}^{S}$ is palindromic if and only if $P_{w}(t)$ is palindromic if and only if $X_{w}$ is rationally smooth. We will say $w$ is a chain if $\left\{v \in \widetilde{W^{S}}\right.$ : $v \leq w\}$ is a totally ordered set.

The outline of the paper is as follows. In Section 2 we establish our basic notation and concepts we hope are familiar to readers. In Section 3, the canonical factorization into segments for elements in $\widetilde{W}^{S}$ is described for all Weyl groups which motivates the definition of the affine partitions. We also state the main theorem giving the bijection from $\widetilde{W}^{S}$ to affine partitions. The type dependent part of the proof of the main theorem is postponed until Sections 5 through 9. In Section 4, we present a new characterization of palindromic elements in terms of affine partitions and generalized Young's lattice. 
After posting the original version of this manuscript on arXiv.org, we learned of the work of Andrew Pruett which also gives canonical reduced expressions for elements in $\widetilde{W}^{S}$ for the simply laced types and characterizes the palindromic elements [26].

\section{Background}

In this section we establish notation and terminology for Weyl groups, affine Weyl groups and partitions. There are several excellent textbooks available which cover this material more thoroughly including $[4,7,15]$ for (affine) Weyl groups and [1, 22, 28, 29] for partitions.

Let $S=\left\{s_{1}, \ldots, s_{n}\right\}$ be the simple generators for $W$ and let $s_{0}$ be the additional generator for $\widetilde{W}$. Let $D$ be the Dynkin diagram for $\widetilde{W}$ as shown on Page 43 . Then the relations on the generators are determined by $D$

$$
\left(s_{i} s_{j}\right)^{m_{i j}}=1
$$

where $m_{i j}=2$ if $i, j$ are not connected in $D$ and otherwise $m_{i j}$ is the multiplicity of the bond between $i, j$ in $D$. A product of generators is reduced if no shorter product determines the same element in $\widehat{W}$.

Let $\ell(w)$ denote the length of $w \in \widetilde{W}$ or the length of any reduced expression for $w$. The Bruhat order on $\widetilde{W}$ is defined by $v \leq w$ if given any reduced expression $w=s_{a_{1}} s_{a_{2}} \cdots s_{a_{p}}$ there exists a subexpression for $v$. Therefore, the cover relation in Bruhat order is defined by

$$
w \text { covers } v \Longleftrightarrow v=s_{a_{1}} s_{a_{2}} \cdots \widehat{s_{a_{i}}} \cdots s_{a_{p}} \text { and } \ell(w)=\ell(v)+1 \text {. }
$$

A partition is a weakly decreasing sequence of positive integers of finite length. By an abuse of terminology, we will also consider a partition to be a weakly decreasing sequence of non-negative integers with a finite number of positive terms. A partition $\lambda=\left(\lambda_{1} \geq \lambda_{2} \geq \lambda_{3} \geq \ldots\right)$ is often depicted by a Ferrers diagram which is a left justified set of squares with $\lambda_{1}$ squares on the top row, $\lambda_{2}$ squares on the second row, etc. For example,

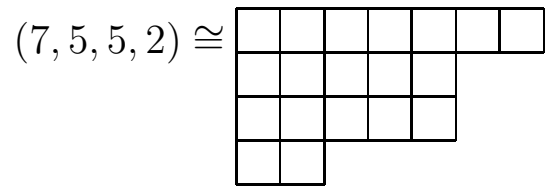

The values $\lambda_{i}$ are called the parts of the partition.

Young's lattice on partitions is an important partial order determined by containment of Ferrers diagrams [22]. In other words, $\mu \subset \lambda$ if $\mu_{i} \leq \lambda_{i}$ for all $i \geq 0$. For example, $(5,5,4) \subset(7,5,5,2)$ in Young's lattice. Young's lattice is a ranked poset with rank function determined by the size of the partition, denoted

$$
|\lambda|=\sum \lambda_{i}
$$


Young's lattice appears as the closure relation on Schubert varieties in the classical Grassmannian varieties [14]. For the isotropic Grassmannians of types $B, C, D$, the containment relation on Schubert varieties is determined by the subposet of Young's lattice on strict partitions, i.e. partitions of the form $\left(\lambda_{1}>\lambda_{2}>\cdots>\lambda_{f}\right)$. This fact follows easily from the signed permutation notation for the Weyl group of types $B / C$. Lascoux [20] has shown that Young's lattice restricted to $(n+1)$-core partitions characterizes the closure relation on Schubert varieties for affine Grassmannians in type $A$. See Section 9 for more details.

\section{Canonical Factorizations and Affine Partitions}

In this section, we will identify a canonical reduced factorization $r(w)$ for each minimal length coset representative $w \in \widetilde{W}^{S}$. The factorizations will be in terms of segments coming from quotients of parabolic subgroups of $\widetilde{W}$. We will use the fact about Coxeter groups that for each $w \in \widetilde{W}$ and each parabolic subgroup $W_{I}=\left\langle s_{i} \mid i \in I\right\rangle$ there exists a unique factorization of $w$ such that $w=u \cdot v, \ell(w)=\ell(u)+\ell(v), u$ is a minimal length element in the coset $u W_{I}$ and $v \in W_{I}$ [4, Prop. 2.4.4]. We will use the notation $u=u_{I}(w)$ and $v=v_{I}(w)$ in this unique factorization of $w$. Let $W^{I}$ denote the minimal length coset representatives for $W / W_{I}$.

Consider the Coxeter graph of an affine Weyl group $\widetilde{W}$ as labeled on Page 43 . The special generator $s_{0}$ is connected to either one or two elements among $s_{1}, \ldots, s_{n}$. If $s_{0}$ is connected to $s_{1}$, call $\widetilde{W}$ a Type I Coxeter group; types $A, C, E, F, G$. If $s_{0}$ is not connected to $s_{1}$, then there is an involution on the Coxeter graph for $\widetilde{W}$ interchanging $s_{0}$ and $s_{1}$ and fixing all other generators. Call these $\widetilde{W}$ Type II Coxeter groups; types $B, D$.

Let $\widetilde{W}$ be a Type I Coxeter group, then the parabolic subgroup generated by $S=$ $\left\{s_{1}, s_{2}, \ldots, s_{n}\right\}$ is the finite Weyl group $W$. Let $J \subset S$ be the subset of generators that commute with $s_{0}$; in particular $s_{1} \notin J$ using our labeling of the generators. Then since $W$ is finite, there are a finite number of minimal length coset representatives in $W^{J}$. For each $j \geq 0$, if there are $k$ elements in $W^{J}$ of length $j$, label these fragments by

$$
F^{1}(j), \ldots, F^{k}(j)
$$

Appending an $s_{0}$ onto the right of each fragment, we obtain elements in $\widetilde{W}^{S}$ called segments. In particular, for each fragment $F^{i}(j)$, fix a reduced expression $F^{i}(j)=$ $s_{a_{1}} s_{a_{2}} \cdots s_{a_{j}}$, and set

$$
\Sigma^{i}(j+1)=s_{a_{1}} s_{a_{2}} \cdots s_{a_{j}} \cdot s_{0} \in \widetilde{W}^{S} .
$$

Now, assume $w \in \widetilde{W}^{S}$ and $w \neq$ id. Let $w^{\prime}=w s_{0}$. Then the unique reduced factorization $w^{\prime}=u_{S}\left(w^{\prime}\right) \cdot v_{S}\left(w^{\prime}\right)$ has the property that $u_{S}\left(w^{\prime}\right)$ is in $\widetilde{W}^{S}$ and $v_{S}\left(w^{\prime}\right) \in W$. In fact, $v_{S}\left(w^{\prime}\right) \in W^{J}$ since $w \in \widetilde{W^{S}}$ and all the generators in $J$ commute with $s_{0}$. Hence, $v_{S}\left(w^{\prime}\right)=$ $F^{i}(j)$ for some $i, j$ so multiplying on the right by $s_{0}$ we have $w=u_{S}\left(w^{\prime}\right) \Sigma^{i}(j+1)$. By induction $u_{S}\left(w^{\prime}\right)$ has a reduced factorization into a product of segments as well. Therefore, 
each $w \in \widetilde{W^{S}}$ has a canonical reduced factorization into a product of segments, denoted

$$
r(w)=\Sigma^{i_{f}}\left(\lambda_{f}\right) \cdots \Sigma^{i_{3}}\left(\lambda_{3}\right) \Sigma^{i_{2}}\left(\lambda_{2}\right) \Sigma^{i_{1}}\left(\lambda_{1}\right)
$$

for some $f \geq 0$ and $\ell(w)=\sum_{j=1}^{f} \lambda_{j}$. Note, $w \in \widetilde{W}^{S}$ may have other reduced factorizations into a product of segments, however, $r(w)$ is unique in the following sense.

Lemma 3. For $w \in \widetilde{W^{S}}$, the canonical reduced factorization $r(w)=\Sigma^{i_{f}}\left(\lambda_{f}\right) \cdots$ $\sum^{i_{2}}\left(\lambda_{2}\right) \sum^{i_{1}}\left(\lambda_{1}\right)$ is the unique reduced factorization of $w$ into a product of segments such that every initial product

$$
\Sigma^{i_{f}}\left(\lambda_{f}\right) \cdots \Sigma^{i_{d+1}}\left(\lambda_{d+1}\right) \Sigma^{i_{d}}\left(\lambda_{d}\right) \quad 1 \leq d \leq f
$$

is equal to $r(u)$ for some $u \in \widetilde{W}^{S}$. Furthermore, every consecutive partial product

$$
\Sigma^{i_{d}}\left(\lambda_{d}\right) \Sigma^{i_{d-1}}\left(\lambda_{d-1}\right) \cdots \Sigma^{i_{c+1}}\left(\lambda_{c+1}\right) \Sigma^{i_{c}}\left(\lambda_{c}\right) \quad 1 \leq c \leq d \leq f
$$

is equal to $r(v)$ for some $v \in \widetilde{W^{S}}$.

Proof. The first claim follows by induction from the uniqueness of the factorization $w=$ $u_{S}\left(w^{\prime}\right) \cdot v_{S}\left(w^{\prime}\right) s_{0}$. To prove the second claim, it is enough to assume $c=1$ by induction. Let $v=\Sigma^{i_{d}}\left(\lambda_{d}\right) \cdots \Sigma^{i_{1}}\left(\lambda_{1}\right)$ as an element of $\widetilde{W}$. Then $v \in \widetilde{W}^{S}$ since $w \in \widetilde{W^{S}}$, and the product $\Sigma^{i_{d}}\left(\lambda_{d}\right) \cdots \Sigma^{i_{2}}\left(\lambda_{2}\right) \sum^{i_{1}}\left(\lambda_{1}\right)$ must be a reduced factorization of $v$ since $r(w)$ is a reduced factorization. Assume by induction on the number of segments in the product that $\Sigma^{i_{d}}\left(\lambda_{d}\right) \cdots \Sigma^{i_{2}}\left(\lambda_{2}\right)=r(u)$ for some $u \in \widetilde{W^{S}}$. Then, by the uniqueness of the factorization $r(v)$, we must have $\Sigma^{i_{d}}\left(\lambda_{d}\right) \cdots \Sigma^{i_{1}}\left(\lambda_{1}\right)=r(v)$.

Remark 4. Observe that the canonical factorization into segments used above extends to any Coxeter system $(W, S)$ with $s_{0}$ replaced by any $s_{i} \in S$ and $J$ replaced by the set $\left\{s_{j}: j \neq i\right.$ and $\left.s_{i} s_{j}=s_{j} s_{i}\right\}$. However, for types $B$ and D, Theorem 8 doesn't hold using this factorization. By using the involution interchanging $s_{0}$ and $s_{1}$ in Type II Weyl groups, we can identify another canonical factorization with shorter segments and all the nice partition properties as with Type I Weyl groups.

Assume $\widetilde{W}$ is a Type II affine Weyl group with generators labeled as on Page 43 . Let $J=\left\{s_{2}, s_{3}, \ldots, s_{n}\right\}$. Note, the parabolic subgroups generated by $S=\left\{s_{1}, s_{2}, s_{3}, \ldots, s_{n}\right\}$ and $S^{\prime}=\left\{s_{0}, s_{2}, s_{3}, \ldots, s_{n}\right\}$ are isomorphic finite Weyl groups. Since $\widetilde{W}_{S^{\prime}}$ is a finite Weyl group, $\left(\widetilde{W}_{S^{\prime}}\right)^{J}$ is finite. For each $j \geq 0$, if there are $k$ elements in $\left(\widetilde{W}_{S^{\prime}}\right)^{J}$ of length $j$, label these 0 -segments by

$$
\Sigma_{0}^{1}(j), \ldots, \Sigma_{0}^{k}(j)
$$

and fix a reduced expression for each one. Similarly, there are a finite number of elements in $W^{J}=\left(\widetilde{W}_{S}\right)^{J}$. For each $j \geq 0$, if there are $k$ minimal length elements of $W^{J}$ of length $j$, label these 1-segments by

$$
\Sigma_{1}^{1}(j), \ldots, \Sigma_{1}^{k}(j)
$$


We will assume each 0,1-pair of segments is labeled consistently so $\Sigma_{1}^{i}(j)$ and $\Sigma_{0}^{i}(j)$ have reduced expressions that differ only in the rightmost generator.

By construction, every $\Sigma_{0}^{i}(j)$ is a minimum length coset representative for $\widetilde{W} / W=$ $\widetilde{W} / W_{S}$ and every $\Sigma_{1}^{i}(j)$ is a minimum length coset representative for $\widetilde{W} / W_{S^{\prime}}$. Let $w \in$ $\widetilde{W}^{S}$, then the unique reduced factorization $w=u_{S^{\prime}}(w) \cdot v_{S^{\prime}}(w)$ has the property that $u_{S^{\prime}}(w) \in \widetilde{W^{S^{\prime}}}$ and $v_{S^{\prime}}(w) \in W_{S^{\prime}}$. In fact, since $w \in \widetilde{W^{S}}$, then $v_{S^{\prime}}(w) \in\left(W_{S^{\prime}}\right)^{J}$ so $v_{S^{\prime}}(w)=\Sigma_{0}^{i}(j)$ for some $i, j$. Similarly, if $y \in \widetilde{W^{S^{\prime}}}$, then $y$ has a unique factorization $y=u_{S}(y) \cdot v_{S}(y)$ where $u_{S}(y) \in \widetilde{W}^{S}$ and $v_{S}(y)=\Sigma_{1}^{i}(j)$ for some $i, j$. Therefore, by induction each $w \in \widetilde{W}^{S}$ has a canonical reduced factorization into a product of alternating 0,1-segments, denoted

$$
r(w)=\cdots \Sigma_{0}^{c_{3}}\left(\lambda_{3}\right) \Sigma_{1}^{c_{2}}\left(\lambda_{2}\right) \Sigma_{0}^{c_{1}}\left(\lambda_{1}\right)
$$

which is unique in the sense of Lemma 3 but where all the consecutive partial products correspond with minimum length coset representatives in $\widetilde{W^{S}}$ or $\widetilde{W^{S}}$ according to their rightmost factor. Note, each $r(w)$ is the product of a finite number of segments, say $f$ of them, and $\ell(w)=\sum_{j=1}^{f} \lambda_{j}$. Note further that the subscripts in (5) are forced to start with 0 on the right and then alternate between 0 and 1. Hence the subscripts can easily be recovered if we omit them and simply use the same notation as in (2).

In both Type I and Type II affine Weyl groups, Lemma 5 below shows that if $r(w)$ factors into segments of lengths $\lambda_{1}, \lambda_{2}, \ldots, \lambda_{f}$ as in (2) or (5), then the sequence of numbers $\left(\lambda_{1}, \lambda_{2}, \ldots\right)$ is a partition of $\ell(w)$ i.e. $\lambda_{1} \geq \lambda_{2} \geq \lambda_{3} \geq \ldots \geq 0$ and $|\lambda|=\sum \lambda_{i}=\ell(w)$. If the segments all have unique lengths, then we can recover $w$ from the partition $\lambda$ by multiplying the corresponding segments in reverse order. However, when there are multiple segments of the same length we will need to allow the parts of the partitions to be "colored" to be able to recover $w$ from the colored partitions. The colors of the parts of a colored partition will be denoted by superscripts. For example, $\left(5^{1}, 5^{1}, 4^{2}, 3^{6}, 1^{2}\right)$ corresponds with the partition $(5,5,4,3,1)$ and the exponents determine the coloring of this partition. Colored partitions are only needed in types $A, D, E, F$. Some colored partitions cannot occur in each type. The rules for determining the allowed colored partitions come from identifying which products of pairs of segments are minimal length coset representatives and which are not.

We will say that $\left(i^{a}, j^{b}\right)$ is an allowed pair if

$$
\Sigma^{a}(i) \cdot \Sigma^{b}(j) \in \widetilde{W^{S}} \text { and } \ell\left(\Sigma^{a}(i) \cdot \Sigma^{b}(j)\right)=i+j .
$$

The following two lemmas describe how segments and allowed pairs relate to the left weak order on $\widetilde{W}^{S}$.

Lemma 5. If $\left(i^{a}, j^{b}\right)$ is an allowed pair, then the following hold:

1. In Type I affine Weyl groups, we have $\Sigma^{a}(i) \leq \Sigma^{b}(j) \in \widetilde{W}^{S}$ in the left weak order on $\widetilde{W}^{S}$.

2. In Type II affine Weyl groups, then $\Sigma_{0}^{a}(i) \leq \Sigma_{0}^{b}(j)$ and $\Sigma_{1}^{a}(i) \leq \Sigma_{1}^{b}(j)$ in left weak order. 
Thus, in either case, if $\left(i^{a}, j^{b}\right)$ is an allowed pair, then $i \leq j$. Furthermore, if in addition $i=j$ then $a=b$.

Proof. The statements (1) and (2) in the lemma will follow by observation in each type once we have identified the segments in later sections. Assuming $W$ is Type I and $\Sigma^{a}(i) \leq \Sigma^{b}(j) \in \widetilde{W}^{S}$ in the left weak order then it follows that $i \leq j$ since $\ell\left(\Sigma^{a}(i)\right)=i$ and $\ell\left(\Sigma^{b}(j)\right)=j$. Furthermore, the only way two elements of the same length can be comparable in the weak order is if they are equivalent, hence $i=j$ implies $\Sigma^{a}(i)=\Sigma^{b}(j)$ which implies $a=b$. The analogous statements hold for the Type II case.

Remark 6. It would be nice to have a type-independent proof of Lemma 5 and to know to what extent this statement holds for all Coxeter groups.

Lemma 7. If $u \in \widetilde{W}^{S}, u \neq i d$, and $u<\Sigma^{a}(i)$ in left weak order, then $u$ is a segment itself, say $u=\Sigma^{c}(h)$. Thus, if $\left(i^{a}, j^{b}\right)$ is an allowed pair, then $\left(h^{c}, j^{b}\right)$ is also an allowed pair.

Note, $\left(h^{c}, i^{a}\right)$ may or may not be an allowed pair if $\Sigma^{c}(h)<\Sigma^{a}(i)$.

Proof. To prove the first part of the statement, note that the set of segments form a lower order ideal in left weak order. Since $\left(i^{a}, j^{b}\right)$ is an allowed pair and $\Sigma^{c}(h)<\Sigma^{a}(i)$ in left weak order, then $\Sigma^{c}(h) \leq \Sigma^{b}(j)$ in left weak order also by Lemma 5 , interpreted with subscripts in the Type II case. Furthermore, $\Sigma^{c}(h) \cdot \Sigma^{b}(j)$ is a right factor of $\Sigma^{a}(i) \cdot \Sigma^{b}(j) \in$ $\widetilde{W}^{S}$ so $\Sigma^{c}(h) \cdot \Sigma^{b}(j)$ is reduced and in $\widetilde{W}^{S}$, thus, $\left(h^{c}, j^{b}\right)$ is also an allowed pair.

Let $\mathcal{P}$ be the set of affine colored partitions: the set of all partitions $\lambda=\left(\lambda_{1}^{c_{1}}, \ldots, \lambda_{f}^{c_{f}}\right)$ such that each consecutive pair $\left(\lambda_{i+1}^{c_{i+1}}, \lambda_{i}^{c_{i}}\right)$ is an allowed pair for $1 \leq i<f$. Note, the order of the consecutive pairs is backwards to the order they appear in $\lambda$. Observe that for every $w \in \widetilde{W}^{S}$, the pairwise consecutive segments in $r(w)$ must correspond with allowed pairs by Lemma 3. Therefore, we can define a map

$$
\begin{aligned}
\pi: \widetilde{W}^{S} & \longrightarrow \mathcal{P} \\
w & \mapsto \lambda
\end{aligned}
$$

if $r(w)=\Sigma^{c_{f}}\left(\lambda_{f}\right) \cdots \Sigma^{c_{2}}\left(\lambda_{2}\right) \Sigma^{c_{1}}\left(\lambda_{1}\right)$ and $\lambda=\left(\lambda_{1}^{c_{1}}, \lambda_{2}^{c_{2}}, \ldots, \lambda_{f}^{c_{f}}\right)$.

Theorem 8. Let $W$ be any Weyl group and $\widetilde{W}$ be the corresponding affine Weyl group. Then $\pi: \widetilde{W}^{S} \rightarrow \mathcal{P}$ is a length preserving bijection.

The theorem above is closely related to theorems of Lam, Lapointe, Morse and Shimozono $[19,17]$ which have been useful in the formulation of Pieri type rules for the cohomology ring of the affine Grassmannian in type $A_{n}$. The same bijection in different language has been used by Eriksson-Eriksson [12] and Reiner [27] to obtain partition identities related to these bijections in types $B, C, D$. See also Bousquet-Mélou and Eriksson $[8,9,10]$ to relate $\widetilde{W}^{S}$ with the lecture hall partitions. More recently Lam-ShimozonoSchilling [18] and Littig-Mitchell [21] have used similar factorizations in various types. Our work provides a more general context in which the affine colored partitions are related to $\widetilde{W}^{S}$ and the affine Grassmannians. 
Proof. Observe that the map $\pi$ is automatically injective since $r(v)=r(w)$ implies $v=w$. Furthermore, the inverse map sending

$$
\lambda=\left(\lambda_{1}^{c_{1}} \geq \lambda_{2}^{c_{2}} \geq \lambda_{3}^{c_{3}} \geq \ldots\right) \mapsto \begin{cases}\ldots \Sigma^{c_{3}}\left(\lambda_{3}\right) \Sigma^{c_{2}}\left(\lambda_{2}\right) \Sigma^{c_{1}}\left(\lambda_{1}\right) & \text { Type I } \\ \ldots \Sigma_{0}^{c_{3}}\left(\lambda_{3}\right) \Sigma_{1}^{c_{2}}\left(\lambda_{2}\right) \Sigma_{0}^{c_{1}}\left(\lambda_{1}\right) & \text { Type II. }\end{cases}
$$

determines a well defined expression $\pi^{-1}(\lambda)$ in $\widetilde{W}$. If $\pi^{-1}(\lambda) \in \widetilde{W^{S}}$ and this expression is reduced, then it must be $r\left(\pi^{-1}(\lambda)\right)$ by Lemma 3 . Therefore, after identifying the segments and allowed pairs in each type, the theorem will follow if we prove

For each $k \geq 0$, the number of partitions of $k$ in $\mathcal{P}$ is equinumerous to the number of elements in $\widetilde{W^{S}}$ of length $k$.

This statement can be proved via a partition identity equating Bott's formula (1) and the rank generating function for $\mathcal{P}$ for all types except type $A$.

This verification occurs in Theorem 28 for type $B$, Theorem 41 for type $C$, and Theorem 52 for type $D$. For type $G_{2}$, this partition identity is easy to check by hand. For types $E_{6}, E_{7}, E_{8}, F_{4}$, computer verification of the identity can be used as discussed in Section 8.

In type $A_{n}$ for $n \geq 2$, the generating function for allowed partitions is not as easy to write down in one formula simultaneously for all $n$. Therefore, surjectivity is proved in Theorem 64 using the $(n+1)$-core partitions.

For emphasis, we state the following corollary of Theorem 8 which is a useful tool for the applications. As opposed to multiplication of generators, the corollary says that reduced multiplication of segments is a "local condition".

Corollary 9. Any product of segments $\Sigma^{c_{1}}\left(j_{1}\right) \Sigma^{c_{2}}\left(j_{2}\right) \cdots \Sigma^{c_{k}}\left(j_{k}\right)$ is equal to $r(v)$ for some $v \in \widetilde{W}^{S}$ if and only if for each $1 \leq i<k$ the pair $\left(j_{i}^{c_{i}}, j_{i+1}^{c_{i+1}}\right)$ is an allowed pair.

Given an affine partition in $\mathcal{P}$, say a corner is $\mathcal{P}$-removable if the partition obtained by removing this corner in the Ferrers diagram leaves a partition that is still in $\mathcal{P}$. The set of $\mathcal{P}$-removable corners for any partition will depend on the affine Weyl group type.

In types $B, C, G_{2}$, we will show that the segments have unique lengths so $\mathcal{P}$ is a subset of all partitions with no colors necessary. It is interesting to note the relationship between Bruhat order on $\widetilde{W}^{S}$ and the induced order from Young's lattice on $\mathcal{P}$. The corollary below shows that Bruhat order on $\widetilde{W}^{S}$ contains the covering relations in Young's lattice determined by $\mathcal{P}$-removable corners.

Corollary 10. Let $\mathcal{P}$ be the set of affine partitions in types $B_{n}, C_{n}$ or $G_{2}$. If $\lambda, \mu \in \mathcal{P}$ and $\lambda$ covers $\mu$ in Young's lattice, then $\pi^{-1}(\lambda)$ covers $\pi^{-1}(\mu)$ in the Bruhat order on $\widetilde{W}^{S}$.

Proof. In types $B_{n}, C_{n}$, and $G_{2}$, the segments form a chain in the left weak order, see Equations (8), (18), and (26). If $\lambda, \mu \in \mathcal{P}$ and $\lambda$ covers $\mu$ in Young's lattice, then $\mu$ is obtained by deleting one outside corner square from $\lambda$. Thus $\Sigma\left(\mu_{g}\right) \cdots \Sigma\left(\mu_{2}\right) \Sigma\left(\mu_{1}\right)$ is obtained from 
$\Sigma\left(\lambda_{f}\right) \cdots \Sigma\left(\lambda_{2}\right) \Sigma\left(\lambda_{1}\right)$ by striking out one generator at the beginning of a segment. This expression will be a reduced expression for a minimal length coset representative precisely when the corresponding partition satisfies the conditions to be in $\mathcal{P}$ by Corollary 9. Given that $\mu \in \mathcal{P}$, then $\pi^{-1}(\lambda)=\Sigma\left(\lambda_{f}\right) \cdots \Sigma\left(\lambda_{2}\right) \Sigma\left(\lambda_{1}\right)>\Sigma\left(\mu_{g}\right) \cdots \Sigma\left(\mu_{2}\right) \Sigma\left(\mu_{1}\right)=\pi^{-1}(\mu)$ and $\ell\left(\pi^{-1}(\lambda)\right)=\ell\left(\pi^{-1}(\mu)\right)+1$. So, $\pi^{-1}(\lambda)$ covers $\pi^{-1}(\mu)$ in the Bruhat order on $\widetilde{W}^{S}$.

For types $A, D, E$, and $F$, we define a generalization of Young's lattice on colored partitions as follows. First, a colored part $j^{c}$ covers another part $(j-1)^{d}$ if $\Sigma^{c}(j)$ covers $\Sigma^{d}(j-1)$ in left weak order on $\widetilde{W}$. Second, a colored partition $\lambda=\left(\lambda_{1}^{c_{1}}, \lambda_{2}^{c_{2}}, \ldots\right) \in \mathcal{P}$ covers $\mu=\left(\mu_{1}^{d_{1}}, \mu_{2}^{d_{2}}, \ldots\right) \in \mathcal{P}$ if $\lambda$ and $\mu$ agree in all but one part indexed by $j$, and $\lambda_{j}^{c_{j}}$ covers $\mu_{j}^{d_{j}}$ in the partial order on colored parts.

Corollary 11. If $\lambda, \mu \in \mathcal{P}$ and $\lambda$ covers $\mu$ in the generalized Young's lattice, then $\pi^{-1}(\lambda)$ covers $\pi^{-1}(\mu)$ in the Bruhat order on $\widetilde{W}^{S}$.

Remark 12. The converse to Corollary 11 does not hold. See Example 31.

Question. Is there an alternative partition $\xi(w)$ to associate with each $w \in \widetilde{W}^{S}$ so that $v<w$ in Bruhat order on $\widetilde{W}^{S}$ if and only if $\xi(v) \subset \xi(w)$ outside of type $A$ ? Recall, the core partitions play this role in type A by a theorem of Lascoux [20].

\section{Palindromic Elements}

As a consequence of the bijection between $\widetilde{W}^{S}$ and affine partitions from Theorem 8, the generalized Young's lattice and Corollary 11, we can observe enough relations in Bruhat order to identify all palindromic elements of $\widetilde{W}^{S}$ in terms of affine partitions. For example, any affine partition with two or more $\mathcal{P}$-removable corners cannot correspond with a palindromic element since Bott's formula starts $1+t+\cdots$ in all types. We recall, the palindromic elements have been recently characterized in [2] via the coroot lattice elements. In type $A_{n}$, there are two infinite families of palindromics, first studied by the second author in [24]. This alternative approach has given us additional insight into the combinatorial structure of $\widetilde{W}^{S}$.

Theorem 13. Assume $W$ is not of type $A_{n}$ for $n \geq 2$ or $B_{3}$. Let $w \in \widetilde{W}^{S}$ and say $\pi(w)=\lambda$. Then $w$ is palindromic if and only if the interval $[i d, w]$ in Bruhat order on $\widetilde{W}^{S}$ is isomorphic to the interval $[\emptyset, \lambda]$ in the generalized Young's lattice and the interval $[\emptyset, \lambda]$ is rank symmetric.

Remark 14. Say $w \in \widetilde{W}^{S}$ is $Y$ B-nice if the interval $[i d, w]$ in Bruhat order on $\widetilde{W}^{S}$ is isomorphic to the interval $[\emptyset, \lambda]$ in the generalized Young's lattice. Say $w$ is YBpalindromic if $w$ is $Y B$ - nice and the interval $[\emptyset, \lambda]$ is rank symmetric. So outside of type $B_{3}$ and $A_{n}$ for $n \geq 2, Y B$-palindromic and palindromic are equivalent. In type $B_{3}$, there is one palindromic element which is not $Y B$-nice namely $w=s_{3} s_{2} s_{0} s_{3} s_{2} s_{1} s_{3} s_{2} s_{0}$. In type $A_{n}$ for $n \geq 2$ the spiral elements which are not closed parabolic orbits are palindromic but not YB-nice. See Section 9. 
Typically the palindromic elements are indexed by one row shapes and staircase shapes. These elements correspond with chains and closed parabolic orbits. The proof of Theorem 13 for the infinite families will be stated and proved more explicitly in Theorems 35, 46 and 57. After stating some general tools used for the palindromy proofs, we prove Theorem 13 for the exceptional types below to demonstrate the technique of using affine partitions.

Define the branching number $b_{W}$ to be the smallest rank in the Bruhat order on $\widetilde{W}^{S}$ with more than 1 element. By Bott's formula, $b_{W} \geq 2$. It will be shown that if $w \in \widetilde{W}^{S}$ is not palindromic, then symmetry of the Poincaré polynomial $P_{w}(t)$ always fails in the first $b_{W}-1$ coefficients for the exceptional types. For some of the other types, we must look further down in Bruhat order.

Let $m_{W}$ be the maximum number of coefficients one must check for all $w \in \widetilde{W}^{S}$ to insure that $P_{w}(t)$ is not palindromic if $w$ is not palindromic. Hence, $m_{W}$ is defined to be the minimum number so that $w \in \widetilde{W}^{S}$ is palindromic if and only if $P_{w}(t)=\sum_{i=0}^{\ell(w)} a_{i} t^{i}$ and $a_{i}=a_{\ell(w)-i}$ for all $1 \leq i \leq m_{W}$. We will show that $m_{W}$ is always bounded by $n$ but can be significantly smaller.

Theorem 15. The number $m_{W}$ for $\widetilde{W}^{S}$ is determined as follows:

\begin{tabular}{|l|c|}
\hline type & $m_{W}$ \\
\hline \hline$A_{1}, n=1$ & 0 \\
$A_{n}, n \geq 2$ & 2 \\
$B_{n}, n=3$ & 2 \\
$B_{n}, n \geq 4$ & 4 \\
$C_{n}, n \geq 2$ & 2 \\
$D_{n}, n \geq 4$ & $n-2$ \\
$E_{6}$ & 3 \\
$E_{7}$ & 4 \\
$E_{8}$ & 6 \\
$F_{4}$ & 4 \\
$G_{2}$ & 4 \\
\hline
\end{tabular}

This theorem will be proved after introducing the segments in each type.

Remark 16. Computationally, the fact that $m_{W}$ is constant in most cases is very useful. This means that an efficient algorithm exists to verify that an element is not palindromic which does not require one to build up the entire Bruhat interval below an element $w \in \widetilde{W}^{S}$ (which takes an exponential amount of time in terms of the length of the element). For example, in type $F_{4}$ it suffices to choose a single reduced expression for $w$ and consider subsequences with at most 4 generators removed. This leads to an $O\left(\ell(w)^{4}\right)$ algorithm.

Remark 17. The number $m_{W}$ bounds the degree of the first nontrivial coefficient in the Kazhdan-Lusztig polynomial $P_{i d, w}(t)$ for $w \in \widetilde{W}^{S}$ by a theorem of Björner and Ekedahl $[5]$. 
We identify the elements of $\widetilde{W^{S}}$ with their corresponding affine partition by Theorem 8 . Therefore, the Bruhat order and the left weak order extends to affine partitions in addition to the generalized Young's lattice. Furthermore, we will abuse notation and denote a colored partition $\left(\lambda_{1}^{c_{1}}, \ldots, \lambda_{k}^{c_{k}}\right)$ simply by $\lambda$ or $\left(\lambda_{1}, \ldots, \lambda_{k}\right)$ when the particular colors are not essential to the argument.

Given an affine partition $\lambda$, we will say $\lambda$ is a thin partition if the interval $[\emptyset, \lambda]$ is rank symmetric in the first and last $b_{W}$ ranks in the generalized Young's lattice. Note, thin is a necessary condition for palindromy by Corollary 11. Furthermore, the following lemma shows that any affine partition whose smallest $k$ parts form a partition that is not thin cannot itself be thin.

Lemma 18. If a colored partition $\left(\lambda_{1}^{c_{1}}, \ldots, \lambda_{k}^{c_{k}}\right) \in \mathcal{P}$ is thin then $\left(\lambda_{2}^{c_{2}}, \ldots, \lambda_{k}^{c_{k}}\right) \in \mathcal{P}$ is thin.

Proof. Suppose $\lambda^{\prime}=\left(\lambda_{2}^{c_{2}}, \ldots, \lambda_{k}^{c_{k}}\right)$ is not thin. Then there exist at least two affine colored partitions $\mu, \nu$ below $\lambda^{\prime}$ in generalized Young's lattice such that $|\mu|=|\nu|>\left|\lambda^{\prime}\right|-b_{W}$. By definition of generalized Young's lattice we must have $\mu_{1}, \nu_{1} \leq \lambda_{2}^{c_{2}}$ in left weak order. Therefore, by Lemma 5 , both $\left(\mu_{1}, \lambda_{1}^{c_{1}}\right)$ and $\left(\nu_{1}, \lambda_{1}^{c_{1}}\right)$ must be allowed pairs. Therefore, concatenating $\lambda_{1}^{c_{1}}$ on the front of $\mu, \nu$ gives two partitions $\mu^{\prime}=\left(\lambda_{1}^{c_{1}}, \mu\right)$ and $\nu^{\prime}=\left(\lambda_{1}^{c_{1}}, \nu\right)$ in $\mathcal{P}$ such that $\mu^{\prime}, \nu^{\prime} \leq \lambda$ in generalized Young's lattice and $\left|\mu^{\prime}\right|=\left|\nu^{\prime}\right|>|\lambda|-b_{W}$, contradicting the fact that $\lambda$ is thin. Hence, $\lambda^{\prime}$ must also be thin.

Observe that $\lambda$ may be thin and still cover two or more elements in Bruhat order; exactly one of the covering relations occurs in the generalized Young's lattice in this case. (See Example 31.) Using similar logic to the proof of Lemma 18, we get the following statement. In Bruhat order, if a colored partition $\lambda=\left(\lambda_{1}, \ldots, \lambda_{k}\right)$ (suppressing the colors) covers two partitions $\mu, \nu$ both with largest part at most $\lambda_{1}$ in left weak order, then any affine partition of the form $\left(\gamma_{1}, \ldots, \gamma_{j}, \lambda_{1}, \ldots, \lambda_{k}\right)=\gamma \cdot \lambda$ also covers two partitions $\gamma \cdot \mu$ and $\gamma . \nu$. Therefore, define an extra thin partition $\lambda$ to be an affine partition that is thin and such that there exists at most one affine partition $\mu$ covered by $\lambda$ in Bruhat order such that the largest part of $\mu$ is less than or equal to the largest part of $\lambda$ in left weak order. Note, extra thin is a necessary condition for palindromy. In fact, this gives an analog of Lemma 18 for extra thin partitions.

Lemma 19. If a colored partition $\left(\lambda_{1}^{c_{1}}, \ldots, \lambda_{k}^{c_{k}}\right) \in \mathcal{P}$ is extra thin then the colored partition $\left(\lambda_{2}^{c_{2}}, \ldots, \lambda_{k}^{c_{k}}\right) \in \mathcal{P}$ is extra thin.

Now we restrict our attention to the exceptional types. Determining the palindromic elements in $\widetilde{W}^{S}$ for the exceptional types follows easily from the affine partitions, the generalized Young's lattice and Lemma 20. For each type there are relatively few palindromics: 8 in $G_{2}, 9$ in $F_{4}$, and 11 in $E_{6,7,8}$. In types $G_{2}$ and $F_{4}$ the palindromics are precisely the chains: elements whose principal lower order ideal is a chain in Bruhat order so $P_{w}(t)=1+t+\cdots+t^{\ell(w)}$. In each of types $E_{6,7,8}$, in addition to the chains there is one palindromic whose Poincaré polynomial is similar to the longest single row in type 
$D$. Plus in types $E_{6,7}$ there are 2 additional Poincaré polynomials that occur. In $E_{6}$, two elements have the Poincaré polynomial

$$
\left(t^{10}+t^{9}+t^{8}+t^{6}+t^{5}+t^{4}+t^{2}+t+1\right)\left(t^{6}+t^{3}+1\right) .
$$

In $E_{7}$, one element has the Poincaré polynomial

$$
\left(t^{21}+t^{16}+t^{14}+t^{12}+t^{9}+t^{7}+t^{5}+1\right)\left(t^{6}+t^{5}+t^{4}+t^{3}+t^{2}+t+1\right)
$$

Lemma 20. For all exceptional types, every affine partition with 7 or more parts is not palindromic. In fact, $m_{W} \leq\left(b_{W}-1\right)$.

Proof. Recall that by Lemma 5, every non-empty affine partition covers at least one element in the generalized Young's lattice by removing a corner of the smallest part. So by Corollary 11, given an affine partition $\mu$ with 7 or more parts, we only need to show there exists one element $\nu$ below $\mu$ in Bruhat order which is not comparable to $\mu$ in generalized Young's lattice and $|\mu|-|\nu|<b_{W}$.

By definition, any affine partition which is not extra thin covers two or more elements in Bruhat order so we can restrict our attention to the extra thin elements. The following observations can be made in the exceptional types with computer assistance:

1. The only extra thin affine partitions with 7 parts in any exceptional type occur in $E_{6}, E_{7}$ and $G_{2}$.

2. In $E_{6}, E_{7}$ and $G_{2}$, every extra thin affine partition with 7 parts has largest 4 parts $\left(j^{c}, j^{c}, j^{c}, j^{c}\right)$ (repeats at least 4 times). Furthermore, if $k^{d}$ is any part such that $\left(j^{c}, k^{d}\right)$ is any allowed pair, then either $j^{c}=k^{d}$ or the affine partition $\left(k^{d}, j^{c}\right)$ has two $\mathcal{P}$-removable corners.

3. The repeated parts $j^{c}$ occurring in every extra thin affine partition with 7 parts described in Part (2) have the property that below $\left(j^{c}, j^{c}, j^{c}, j^{c}\right)$ in Bruhat order there exists some affine partition $\lambda$ whose parts are all weakly larger than $j^{c}$ in the left weak order and $|\lambda|>4 j-b_{W}$.

Using these observations we complete the proof. In types $E_{8}$ and $F_{4}$ every affine partition with 7 or more parts is not extra thin by the first observation and Lemma 19 . So, assume the type is $E_{6}, E_{7}$ or $G_{2}$. By the second observation, the only colored part which is allowed to extend an extra thin affine partition with 7 or more parts to another extra thin affine partition is another copy of its largest part. Therefore, every extra thin affine partition $\mu$ with at least 7 parts has its largest part repeated at least 4 times. Say $\mu=\left(j^{c}, j^{c}, j^{c}, j^{c}, \mu_{5}^{c_{5}}, \ldots, \mu_{k}^{c_{k}}\right)$ and say $\lambda=\left(\lambda_{1}^{d_{1}}, \ldots, \lambda_{p}^{d_{p}}\right)$ is the affine partition below $\left(j^{c}, j^{c}, j^{c}, j^{c}\right)$ in Bruhat order appearing in the third observation. Then $\nu=\left(\lambda_{1}^{d_{1}}, \ldots, \lambda_{p}^{d_{p}}, \mu_{5}^{c_{5}}, \ldots, \mu_{k}^{c_{k}}\right)$ must be an affine partition by Lemma 5 and the definition of $\mathcal{P}$. Furthermore, $\nu$ is not in the interval below $\mu$ in the generalized Young's lattice but $\nu<\mu$ in Bruhat order and $|\nu|>|\mu|-b_{W}$. Hence $\mu$ fails to be palindromic by the $\left(b_{W}-1\right)^{t h}$ coefficient. 
Remark 21. Looking closely at the data, one sees that in fact every affine partition in $E_{7}, E_{8}$ and $F_{4}$ with 7 or more parts covers at least two elements in Bruhat order. In $E_{6}$ and $G_{2}$, there exist an infinite number of extra thin non-palindromic elements which cover a single element in Bruhat order and only fail to be palindromic on the second and fourth coefficient respectively. In type $E_{6}$ there are two segments of length 12 which can repeat an arbitrary number of times to get such elements. In type $G_{2}$, there is a unique segment of length 5 , and the affine partition $(5,5, \ldots, 5)$ with $k \geq 2$ parts has this property.

Theorem 22. In each exceptional type we have:

1. No affine partition $\lambda \in \mathcal{P}$ with 4 or more parts corresponds with a palindromic $w \in W^{S}$. In fact, the unique palindromic element with 3 parts occurs in $E_{7}$.

2. A finite computer search over all extra thin affine partitions with at most 3 parts suffices to identify all palindromic elements in $\mathcal{P}$, or equivalently in $\widetilde{W}^{S}$.

3. We have $m_{W}=b_{W}-1$.

Proof. Note, every element which is not extra thin fails to be palindromic by depth $b_{W}-1$ by definition. Therefore, by Lemma 20 , we only needed to verify these statements for extra thin affine partitions with at most 6 parts which is efficient to check by computer. In each case, it was verified that every non-palindromic fails to be palindromic by depth $b_{W}-1$, and furthermore, there is at least one non-palindromic element that is palindromic up to the $\left(b_{W}-2\right)^{n d}$ coefficient.

Remark 23. We note that the proof of Theorem 13 in the exceptional types now follows from Theorem 22 simply by verifying that for every palindromic element the corresponding Bruhat interval is isomorphic to the interval in generalized Young's lattice.

\section{Type $B$}

In this section we prove Theorem 8 and Theorem 13 for type $B_{n}, n \geq 3$. We begin by identifying a family of partitions $\mathcal{P}\left(B_{n}\right)$ with a length preserving bijection to $\widetilde{W}^{S}$. Then, we identify the segments in type $B$ and the allowed pairs corresponding to these segments. It follows immediately from the list of allowed pairs that $\mathcal{P}\left(B_{n}\right)$ are the affine partitions. Finally we use the explicit description of the segments in this type to identify the affine partitions corresponding with palindromic elements in $\widetilde{W}^{S}$.

Let $\mathcal{P}\left(B_{n}\right)$ be the set of partitions whose parts are bounded by $2 n-1$ and all the parts of length strictly less than $n$ are strictly decreasing. Note, all parts in these partitions have the same color. Therefore, the generating function for such partitions is

$$
G_{B_{n}}(x)=\frac{(1+x)\left(1+x^{2}\right) \cdots\left(1+x^{n-1}\right)}{\left(1-x^{n}\right)\left(1-x^{n+1}\right) \cdots\left(1-x^{2 n-1}\right)}
$$

THE ELECTRONiC JOURNAL OF COMBINATORICS 16(2) (2009), \#R18 
Lemma 24. We have the following generating function identity with Bott's formula from (1):

$$
G_{B_{n}}(x)=\frac{1}{(1-x)\left(1-x^{3}\right)\left(1-x^{5}\right) \cdots\left(1-x^{2 n-1}\right)} .
$$

Proof. Apply induction on $n$.

Consider the Coxeter graph of $\widetilde{B}_{n}$ on Page 43 . In the language of Section $3, \widetilde{B}_{n}$ is a Type II Coxeter graph. Following (3) and (4), for $1 \leq j \leq 2 n-1$, set

$$
\Sigma_{1}(j)= \begin{cases}s_{j} \ldots s_{3} s_{2} s_{1} & 1 \leq j \leq n \\ s_{2 n-j} \cdots s_{n-1} s_{n} s_{n-1} \cdots s_{4} s_{3} s_{2} s_{1} & n<j \leq 2 n-1 .\end{cases}
$$

Similarly, replacing all the $s_{1}$ 's in $\Sigma_{1}(j)$ with $s_{0}$ 's, set

$$
\Sigma_{0}(j)= \begin{cases}s_{0} & j=1 \\ s_{j} \ldots s_{3} s_{2} s_{0} & 1<j \leq n \\ s_{2 n-j} \ldots s_{n-1} s_{n} s_{n-1} \ldots s_{3} s_{2} s_{0} & n<j \leq 2 n-2 \\ s_{0} s_{2} s_{3} \ldots s_{n-1} s_{n} s_{n-1} \ldots s_{3} s_{2} s_{0} & j=2 n-1\end{cases}
$$

Using the signed permutation description of the finite Weyl group of type $B_{n}$, one can verify that these segments are all the minimal length coset representatives for $W / W_{J}$ and $\widetilde{W}_{S^{\prime}} / W_{J}$ respectively. See [4, Chapter 8] for a detailed description of this notation.

Lemma 25. We have the following commutation rules for $s_{i} \cdot \Sigma_{1}(j)$ for all $1 \leq i \leq n$ and $1 \leq j \leq 2 n-1$ :

$$
s_{i} \cdot \Sigma_{1}(j)= \begin{cases}\Sigma_{1}(j) \cdot s_{i} & 1 \leq j<i-1 \text { or } 2 n-i<j \leq 2 n-1 \\ \Sigma_{1}(j+1) & j=i-1 \text { or } j=2 n-i-1 \\ \Sigma_{1}(j-1) & j=i \text { or } j=2 n-i \\ \Sigma_{1}(j) \cdot s_{i+1} & 1 \leq i<j<2 n-i-1,\end{cases}
$$

and for $i=0$ and $j=1$ we have

$$
s_{0} \Sigma_{1}(1)=s_{0} s_{1}=s_{1} s_{0}=\Sigma_{1}(1) s_{0} .
$$

Similar commutation rules for $s_{i} \Sigma_{0}(j)$ for all $0 \leq i \leq n$ and $1 \leq j \leq 2 n-1$ are obtained from (10) by interchanging the roles of $s_{1}$ and $s_{0}$.

Proof. These follow directly from the commutation relations among the generators determined by the Dynkin diagram.

Lemma 26. We have the following product rules for segments in type $B_{n}$ :

1. For $1 \leq j<n$,

$$
\Sigma_{1}(j) \Sigma_{0}(j)=\Sigma_{1}(j-1) \Sigma_{0}(j) \cdot s_{1}
$$

THE ELECTRONIC JOURNAL OF COMBINATORICS 16(2) (2009), \#R18 
2. For $n \leq j \leq 2 n-1$,

$$
\Sigma_{1}(j+1) \Sigma_{0}(j)=\Sigma_{1}(j) \Sigma_{0}(j) \cdot s_{1} .
$$

Proof. If $j=1$, then Statement 1 holds since $s_{0}$ and $s_{1}$ commute. Assume $2 \leq j<n$. By Lemma 25 , we have $\Sigma_{1}(j) s_{i}=s_{i-1} \Sigma_{1}(j)$ for all $2 \leq i \leq j$ so

$$
\begin{aligned}
\Sigma_{1}(j) \cdot \Sigma_{0}(j) & =\Sigma_{1}(j) \cdot s_{j} s_{j-1} \cdots s_{2} s_{0} \\
& =s_{j-1} \cdots s_{2} s_{1} \cdot \Sigma_{1}(j) \cdot s_{0} \\
& =\Sigma_{1}(j-1) \cdot s_{j} s_{j-1} \cdots s_{2} s_{1} \cdot s_{0} \\
& =\Sigma_{1}(j-1) \cdot s_{j} s_{j-1} \cdots s_{2} \cdot s_{0} s_{1} \\
& =\Sigma_{1}(j-1) \cdot \Sigma_{0}(j) \cdot s_{1} .
\end{aligned}
$$

Statement 2 holds by a similar argument.

Corollary 27. We have the following product rules for segments corresponding with all pairs $(j, k) \notin \mathcal{P}\left(B_{n}\right)$ with $1 \leq j, k \leq 2 n-1$ :

$$
\Sigma_{1}(k) \cdot \Sigma_{0}(j)= \begin{cases}\Sigma_{1}(j-1) \cdot \Sigma_{0}(k) \cdot s_{1} & j \leq k<2 n-j \\ \Sigma_{1}(j) \cdot \Sigma_{0}(k-1) \cdot s_{1} & n \leq j<k \text { or } j<2 n-j \leq k .\end{cases}
$$

Proof. This follows from Lemma 25 and Lemma 26.

Recall, $\mathcal{P}$ is the set of allowed partitions for $\widetilde{W}^{S}$ and $\pi: \widetilde{W}^{S} \longrightarrow \mathcal{P}$ was defined in (6).

Theorem 28. In type $B_{n}$, we have $\mathcal{P}=\mathcal{P}\left(B_{n}\right)$ and $\pi: \widetilde{W}^{S} \longrightarrow \mathcal{P}$ is a length preserving bijection.

Proof. We have already shown in the proof of Theorem 8 that $\pi$ is a length preserving injection. Since there is a length preserving bijection from $\widetilde{W}^{S}$ to $\mathcal{P}\left(B_{n}\right)$ by Lemma 24 , we only need to show that $\mathcal{P} \subset \mathcal{P}\left(B_{n}\right)$ to prove the theorem. Therefore, we only need to show that the segments have unique lengths between 1 and $2 n-1$ and that all allowed pairs in type $B_{n}$ are strictly increasing if the larger part has length less than $n$. This follows directly from the definition of the segments and Corollary 27.

\begin{tabular}{|c|c|}
\hline \begin{tabular}{l|l|l}
0 & 2 & 3
\end{tabular} & \begin{tabular}{|l|l|l}
3 & 4 & 3
\end{tabular} \\
\hline & \begin{tabular}{|l|l|l}
3 & 4 & 3 \\
\end{tabular} \\
\hline \begin{tabular}{l|l|l}
0 & 3 \\
\end{tabular} & \begin{tabular}{|l|l|l|}
3 & 4 & 3 \\
\end{tabular} \\
\hline \begin{tabular}{l|l|l}
1 & 2 & 3 \\
0
\end{tabular} & \\
\hline
\end{tabular}

The counting argument in the proof above determines the complete set of allowed pairs.

Corollary 29. The product $\Sigma_{1}(j) \cdot \Sigma_{0}(k) \in \widetilde{W}^{S}$ and $\ell\left(\Sigma_{1}(j) \cdot \Sigma_{0}(k)\right)=j+k$ if and only if $1 \leq j<k \leq 2 n-1$ or $n \leq j=k \leq 2 n-1$.

Example 30. If $n=4$, the partition $(7,5,5,3,1) \in \mathcal{P}\left(B_{n}\right)$ corresponds with $s_{0} \cdot s_{3} s_{2} s_{1}$. $s_{3} s_{4} s_{3} s_{2} s_{0} \cdot s_{3} s_{4} s_{3} s_{2} s_{1} \cdot s_{0} s_{2} s_{3} s_{4} s_{3} s_{2} s_{0}$. Pictorially, we have 
where the corresponding reduced expression is read along the rows from right to left, bottom to top. Note, the 0's and 1's alternate in the first column starting with a 0 in the top row since $B_{n}$ is a Type II Coxeter group. The other columns contain their column number up to column $n$, for $n<j<2 n-1$ column $j$ contains $2 n-j$, and column $2 n-1$ again alternates from 0 to 1 starting from the top.

Recall from Corollary 10, that if both $\lambda, \mu \in \mathcal{P}\left(B_{n}\right)$ and $\lambda$ covers $\mu$ in Young's lattice then $\pi^{-1}(\lambda)$ covers $\pi^{-1}(\mu)$ in Bruhat order on $\widetilde{W}^{S}$. However, below we give an example where the converse does not hold.

Example 31. If $n=4$, the partition $(5,2,1) \in \mathcal{P}\left(B_{n}\right)$ corresponds with $s_{0} \cdot s_{2} s_{1} \cdot s_{3} s_{4} s_{3} s_{2} s_{0}$ which covers $s_{0} s_{2} s_{3} s_{4} s_{3} s_{2} s_{0}=\Sigma_{0}(7)$ by deleting $s_{1}$. Pictorially,

$$
(5,2,1) \cong \begin{array}{l|l|l|l|l}
\hline 0 & 2 & 3 & 4 & 3
\end{array} \quad \text { covers } \quad(7) \cong \begin{array}{ll|l|l|l|l|l|l|}
0 & 2 & 3 & 4 & 3 & 2 & 0 \\
\hline 1 & 2 & &
\end{array} \quad \text {. }
$$

Therefore, in Bruhat order $(5,2,1)$ covers $(7)$ even though $(5,2,1)$ is not comparable to (7) in Young's lattice.

With more work, we can obtain all of the elements below $w \in \widetilde{W}^{S}$ in Bruhat order by looking at $r(w)$, knocking out one generator at a time, and using the commutation relations in Lemma 25 and Corollary 27 to identify a canonical segmented expression for the new element. In fact, in practice it is easy to see that knocking out most elements will lead to products for non-minimal length coset representatives or non-reduced expressions.

Corollary 32. If $v<w$ in Bruhat order on $\widetilde{W}^{S}$ and $\ell(w)=\ell(v)+1$, then one of the following must hold:

1. The partition $\pi(v)$ is obtained from $\pi(w)$ by removing a single outer corner square so that the remaining shape is in $\mathcal{P}$.

2. The partition $\pi(v)$ is obtained from $\pi(w)$ by removing a single inside square $s$ in row $i$ of the Ferrers diagram so that the generators corresponding to the squares to the right of square s all commute up to join onto the end of an existing row above row $i$, and furthermore, the resulting product of segments can be put into canonical form using the commutation relations in Lemma 25 and Lemma 26 in such a way that they correspond with a partition in $\mathcal{P}$ with one fewer square.

Lemma 33. If $\lambda \in \mathcal{P}\left(B_{n}\right)$ and $\lambda_{1}<n$, then the interval in Bruhat order between id and $\pi^{-1}(\lambda)$ is isomorphic as posets to the interval from the empty partition to $\lambda$ in Young's lattice on strict partitions.

The lemma above is "well-known" in the following sense. If $\lambda_{1}<n$, then $s_{n}$ does not appear in any reduced expression for $\pi^{-1}(\lambda)$. Thus, the interval from id and $\pi^{-1}(\lambda)$ in Bruhat order is isomorphic to an interval in Grassmannian quotient of the finite Weyl group of type $D_{n-1}$ modulo $I=\left\{s_{1}, \ldots, s_{n-1}\right\}$. Using Proctor's criterion for Bruhat order in $D_{n-1}[25]$ or [4, Theorem 8.2.8], the lemma now follows. It can also be proven directly using the type $B$ segments. 
Lemma 34. If $\lambda \in \mathcal{P}\left(B_{n}\right)$ is a single row $(\lambda=(j))$, then the interval in Bruhat order between id and $\pi^{-1}(\lambda)$ is isomorphic as posets to the interval from the empty partition to $\lambda$ in Young's lattice. In particular, this interval is a totally ordered chain.

Proof. If $\lambda$ is a single row of length $j$, then $r\left(\pi^{-1}(\lambda)\right)=\Sigma_{0}(j)$. Observe directly from the relations on the generators that deleting any but the leftmost generator from $\Sigma_{0}(j)$ either leaves a non-reduced expression or a non-minimal length coset representative by definition.

Consider the Bruhat order on $\widetilde{W^{S}}$ expressed in terms of partitions in $\mathcal{P}\left(B_{n}\right)$ for $n \geq 3$. By Lemma 33, the smallest 4 ranks of the Bruhat Hasse diagram are determined by Young's lattice on strict partitions:

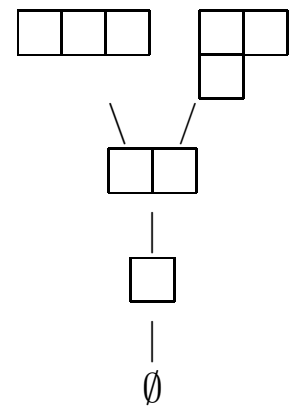

Theorem 35. Let $\widetilde{W}$ be the affine Weyl group of type $B_{n}$ and let $w \in \widetilde{W}^{S}$. For $n \geq 4$, w is palindromic if and only if $\pi(w)$ is a one row shape $(j)$ with $0 \leq j<2 n$ or a staircase shape $(k, k-1, k-2, \ldots, 1)$ for $1<k<n$. For $n=3$, $w$ is palindromic if and only if $\pi(w)$ is a one row shape or the staircase shape $(2,1)$ or the square $(3,3,3)$.

Remark 36. The number of palindromic elements in $\widetilde{W}^{S}$ for type $B_{n}$ is therefore 8 for $n=3$ and $3 n-2$ for $n \geq 4$. The one row shapes with $n \leq j \leq 2 n-2$ and the $(3,3,3)$ shape in $n=3$ correspond with rationally smooth Schubert varieties which are not actually smooth. All others are closed parabolic orbits, hence they are smooth by Theorem 1.

Proof. By definition, $w$ is palindromic if and only if its Poincaré polynomial $P_{w}(t)=$ $\sum_{v \leq w} t^{\ell(v)}$ is palindromic. By Lemma 34, we know that $P_{w}(t)=1+t+\cdots+t^{j}$ if $\pi(w)$ is a one row shape, which is clearly palindromic. By Lemma 33, if $\pi(w)$ is a staircase shape $(k, k-1, k-2, \ldots, 1)$ for $1<k<n$ and $n \geq 3$, then

$$
P_{w}(t)=\prod_{j=1}^{k}\left(1+t^{j}\right)
$$

which is easily seen to be palindromic by induction. In the case $n=3$, one can verify that for $w=s_{3} s_{2} s_{0} \cdot s_{3} s_{2} s_{1} \cdot s_{3} s_{2} s_{0}$ corresponding with $\pi(w)=(3,3,3)$, the Poincaré polynomial for $X_{w}$ is

$$
\begin{aligned}
P_{w}(t) & =t^{9}+t^{8}+t^{7}+2 t^{6}+2 t^{5}+2 t^{4}+2 t^{3}+t^{2}+t^{1}+1 \\
& =(1+t)\left(1+t^{2}\right)\left(1+t^{3}+t^{6}\right)+t^{4}+t^{5}
\end{aligned}
$$


which is palindromic. ${ }^{2}$

Conversely, we will show that if $\pi(w)$ is not a one row shape or a staircase shape or $(3,3,3)$ in the case $n=3$, then for some $0<j<\ell(w)$ one pair of the coefficients in

$$
P_{w}(t)=\sum_{i=0}^{\ell(w)} a_{i} t^{i}
$$

has the property that $a_{j} \neq a_{\ell(w)-j}$. We can calculate all $a_{j}$ for $0<j \leq n$ by computing the number of strict partitions that fit inside the Ferrers diagram for $\pi(w)$ since there are no repeated parts in any partition in $\mathcal{P}\left(B_{n}\right)$ of size $n$ or less and $\mathcal{P}\left(B_{n}\right)$ contains all such strict partitions. Therefore, in order to show $P_{w}(t)$ is not palindromic, it suffices to show there exists some $1<j \leq n$ such that $a_{\ell(w)-j}$ is bigger than the number of strict partitions below $\pi(w)$ of size $j$.

Assume $\lambda=\pi(w)$ has at least two rows. From (16), we know the branching number $b_{W}=2$ so $a_{1}=a_{2}=1$. If $\lambda$ has two or more $\mathcal{P}$-removable outside corners, then $a_{\ell(w)-1} \geq 2$ so $w$ is not palindromic. Similarly, if $\lambda$ has one $\mathcal{P}$-removable corner and removing it leaves a shape with two $\mathcal{P}$-removable corners, then $a_{\ell(w)-2} \geq 1$ so $w$ is not palindromic. One of these two cases occurs for all partitions $\lambda \in \mathcal{P}\left(B_{n}\right)$ with at least two rows and $\lambda_{1}>n$ or if there exists an $i$ such that $\lambda_{i}-\lambda_{i+1} \geq 2$. Therefore, it remains to show that if $\lambda=(n, n, \ldots, n)=\left(n^{k}\right)$ or $\lambda=\left(n^{k}, n-1, \ldots, 1\right)$ for some $k>1$ then $P_{w}(t)$ is not palindromic excluding the case $\lambda=(3,3,3)$ and $n=3$.

If $\lambda=\left(n^{k}\right)$ then the interval below $\lambda$ in Young's lattice restricted to $\mathcal{P}\left(B_{n}\right)$ is self dual, and hence rank symmetric, since we can pair any $\gamma \subset \lambda$ with its complement inside the rectangle of size $n \times k$. Therefore, for each $k>1$, in order to show $w$ is not palindromic, we simply need to find a single partition $\mu \in \mathcal{P}\left(B_{n}\right)$ such that $\pi^{-1}(\mu)<w$ in Bruhat order, $\mu \not \subset \lambda$ in Young's lattice, and $\ell(\mu) \geq \ell(w)-n$.

Assume first that $1<k<n$. Let $i=n-k$. Then, $w>\pi^{-1}\left(n^{k-1}, n-1\right)$ and deleting the generator in column $i$ on row $k$ in the reduced expression corresponding with $\left(n^{k-1}, n-1\right)$ gives an expression where the leftmost $s_{i+1}$ commutes right past $k-2$ segments of length $n$, increasing its index as it commutes past each segment, to become an $s_{i+1+k-2}=s_{n-1}$ which glues onto the rightmost $\Sigma_{0}(n)$. Similarly, the leftmost $s_{i+2}$ commutes right past $k-3$ segments and glues onto the rightmost $\Sigma_{1}(n)$ to become $\Sigma_{1}(n+$ $1)$, etc for the left most $s_{i+3}, \ldots s_{n-1}$. The remaining expression corresponds with the partition $\mu=\left((n+1)^{k-1}, n-k-1\right) \not \subset\left(n^{k}\right)$, but $v=\pi^{-1}(\mu)<w$ in Bruhat order and $\ell(v)=\ell(w)-2$ so $w$ is not palindromic. For example, if $n=6, k=4$, then if $w=\pi^{-1}(6,6,6,6)$, we have $\mu=(7,7,7,1)$ and $v=\pi^{-1}(\mu)$ is obtained from $\pi^{-1}(6,6,6,5)$ by deleting one more generator and applying commutation relations:

$$
\lambda=\begin{array}{|l|l|l|l|l|l|}
\hline 0 & 2 & 3 & 4 & 5 & 6 \\
\hline 1 & 2 & 3 & 4 & 5 & 6 \\
\hline 0 & 2 & 3 & 4 & 5 & 6 \\
\hline 1 & 2 & 3 & 4 & 5 & 6 \\
\hline
\end{array} \quad>\quad \begin{array}{|l|l|l|l|l|l}
\hline & 2 & 3 & 4 & 5 & 6 \\
\hline 1 & 2 & 3 & 4 & 5 & 6 \\
\hline 0 & 2 & 3 & 4 & 5 & 6 \\
\hline 1 & & 3 & 4 & 5 &
\end{array}=\mid \begin{array}{ll|l|l|l|l|l|}
0 & 2 & 3 & 4 & 5 & 6 & 5 \\
\hline 1 & 2 & 3 & 4 & 5 & 6 & 5 \\
\hline 0 & 2 & 3 & 4 & 5 & 6 & 5 \\
\hline 1 & \multicolumn{1}{|l}{} \\
\hline
\end{array}
$$

\footnotetext{
${ }^{2}$ It is interesting to observe that the interval below $w=\pi^{-1}(3,3,3)$ contains all the elements corresponding with the strict partitions contained in $(3,3,3)$ plus the two additional elements $\pi^{-1}(5)$ and $\pi^{-1}(4)$ in Bruhat order which contribute symmetrically to $P_{w}(t)$.
} 
Assume next that $\lambda=\left(n^{n}\right)$, we claim that $\mu=\left((n+2)^{n-2}\right)$ is a partition in $\mathcal{P}\left(B_{n}\right)$ which is not contained in $\lambda$ such that $\pi^{-1}(\mu)<w$ in Bruhat order and $\ell\left(\pi^{-1}(\mu)\right)=$ $\ell(w)-4$. For $n \geq 4$, this implies $a_{4}<a_{\ell(w)-4}$, so $w$ is not palindromic. To prove the claim, observe that by Lemma 33, $w>\pi^{-1}\left(n^{n-2}, n-1, n-2\right)$. Let $v^{\prime}$ be the element obtained by deleting the generator corresponding to row $n-1$ and column 1 in $\pi^{-1}\left(n^{n-2}, n-1, n-2\right)$. Assume $n$ is even for the sake of notation (the case $n$ odd is the same except for a 0,1 switch). Then, by definition

$$
v^{\prime}=s_{n-2} \cdots s_{2} s_{1} \cdot s_{n-1} \cdots s_{3} s_{2} \cdot \pi^{-1}\left(n^{n-2}\right) .
$$

Note that by Lemma $25, s_{2} \cdot \pi^{-1}\left(n^{n-2}\right)=\pi^{-1}\left(n+1, n^{n-3}\right)$ since the index on the generator $s_{2}$ increases by one each time it commutes with a $\Sigma_{0}(n)$ or $\Sigma_{1}(n)$, after commuting with $n-3$ segments $s_{2}$ becomes $s_{n-1}$ and $s_{n-1} \cdot \Sigma_{0}(n)=\Sigma_{0}(n+1)$. After moving the $s_{2}$, the last $s_{1}$ commutes right as far as possible and becomes an $s_{n-2}$ which glues onto the rightmost segment. Continuing in the same way we can commute all the generators remaining in the expression for $v^{\prime}$ above in the last two rows of $\left(n^{n}\right)$ up to glue onto rows $1, \ldots, n-2$ adding two additional generators to each row, so $\pi^{-1}(\mu)=v<w$.

Similarly, if $\pi(w)$ is any partition in $\mathcal{P}\left(B_{n}\right)$ with $n \geq 4$ of the form $=\left(n^{k}, \lambda_{k+1}, \ldots, \lambda_{m}\right)$ with $k>n$, then $r(w)$ has a right factor of $\pi^{-1}\left(n^{n}\right)$ so as above $w$ is bigger than $v=$ $\pi^{-1}\left((n+2)^{n-2}, n^{k-n}, \lambda_{k+1}, \ldots, \lambda_{m}\right)$ and $\ell(v)=\ell(w)-4$, so $w$ is not palindromic.

If $n=3$, we claim any affine partition $\left(3^{k}, \lambda_{k+1}, \ldots, \lambda_{m}\right)$ is not palindromic for $k>3$. To see this note that $\left(3^{5}\right)$ covers $(4,4,3,3)$, so $\left(3^{k}, \lambda_{k+1}, \ldots, \lambda_{m}\right)$ covers at least 2 elements in Bruhat order, hence is not palindromic. For $k>5$, one may extend this observation by using an argument similar to the proof of Lemma 20. For $k=4$, it is easy to verify the claim on the 4 possible affine partitions. For example, $\left(3^{4}\right)$ covers $(3,3,3,2)$ and $(4,3,3,1)$ chopping out the 3 on the 3rd row. Similarly, $\left(3^{4}, 2\right)$ covers $(4,3,3,3)$ and $(4,4,3,2)$.

Finally, we need to address the case $\lambda=\left(n^{k}, n-1, n-2, \ldots, 2,1\right)$ for $1 \leq k<n$ and $n \geq 4$. We claim $w$ covers two elements of length $\ell(w)-1$ in $\widetilde{W}^{S}$. The first element is obtained by deleting the last row of $\lambda$. The second element, indexed by the partition $\left((n+1)^{k-1}, n, n-1, \ldots, \widehat{k}, \ldots, 2,1\right)$, is obtained from $\lambda$ by deleting the corner square in row $n$ column $k$ to obtain the expression (suppressing the 0,1 subscripts so we don't need to consider the parity of $n)$ :

$$
\Sigma(1) \Sigma(2) \cdots \Sigma(k-1) \Sigma(k-1) \cdot \pi^{-1}\left(n^{k}, n-1, n-2, \ldots, k+1\right)
$$

Now, by Lemma $26, \Sigma_{1}(k-1) \Sigma_{0}(k-1)=\Sigma_{1}(k-2) \Sigma_{0}(k-1) \cdot s_{1}$. Moving the $s_{1}$ on the right up past $n-2$ rows it becomes $s_{n-1}$ by Lemma 25 which glues onto row 1 to become a $\Sigma_{0}(n+1)$. Swapping $s_{1}$ with $s_{0}$ results in the same partition shapes. After rectifying the two segments of length $k-1$ there are two segments of length $k-2$ which rectify by adding an additional generator to row 2, etc. Applying the same technique repeatedly we can rectify the whole expression to obtain an equivalent reduced expression corresponding to the shape obtained from $\lambda$ by removing the corner square from each row from $n$ to row $n+k-1$ and adding one square to rows 1 through $k-1$.

Recall the definition of $m_{W}$ from Section 4.

Corollary 37. For $W$ of type $B_{n}$, we have $m_{W}=4$ for $n \geq 4$ and $m_{W}=2$ for $n=3$. 


\section{Type $C$}

Let $\mathcal{P}\left(C_{n}\right)$ be the set of partitions whose parts are bounded by $2 n$ and the parts of length less than or equal to $n$ are strictly decreasing. The generating function for such partitions is

$$
G_{C_{n}}(x)=\frac{(1+x)\left(1+x^{2}\right) \cdots\left(1+x^{n}\right)}{\left(1-x^{n+1}\right)\left(1-x^{n+2}\right) \cdots\left(1-x^{2 n}\right)}
$$

Lemma 38. We have the following generating function identity with Bott's formula from (1):

$$
G_{C_{n}}(x)=\frac{1}{(1-x)\left(1-x^{3}\right)\left(1-x^{5}\right) \cdots\left(1-x^{2 n-1}\right)} .
$$

Proof. Apply induction on $n$.

The Dynkin diagram of $\widetilde{C}_{n}$ with the extra generator labeled $s_{0}$ is adjacent to $s_{1}$ so $\widetilde{W}^{S}$ will have Type I segments and fragments as described in Section 3. The parabolic subgroup generated by $S=\left\{s_{1}, s_{2}, s_{3} \ldots, s_{n}\right\}$ corresponds with the finite Weyl group of type $C_{n}$. Let $J=\left\{s_{2}, \ldots, s_{n}\right\}$. Then the minimal length coset representatives for $W / W_{J}$ are the fragments

$$
\begin{array}{cl}
\text { id } & \text { for } 1 \leq i \leq n \\
s_{i} \cdots s_{3} s_{2} s_{1} & \text { for } 1 \leq i<n \\
s_{i} \cdots s_{n-1} s_{n} s_{n-1} \cdots s_{3} s_{2} s_{1} &
\end{array}
$$

The fact that these are all the minimum length coset representatives for $W_{I} / W_{J}$ is easily checked using the signed permutation description of the type $C_{n}$ Weyl group elements [4]. We will use the reduced expressions in (17) to define the segments $\Sigma^{i}(j)$. Note, in this case, each word in (17) has unique length, so we will denote $\Sigma^{1}(j)$ by simply $\Sigma(j)$. Set $\Sigma(0)=\mathrm{id}$, and for $1 \leq j \leq 2 n$ set

$$
\Sigma(j)= \begin{cases}s_{j-1} \ldots s_{2} s_{1} s_{0} & 1 \leq j \leq n+1 \\ s_{2 n-j+1} \cdots s_{n-1} s_{n} s_{n-1} \cdots s_{2} s_{1} s_{0} & n+1<j \leq 2 n .\end{cases}
$$

The proofs of the next two lemmas are analogous to Lemmas 25 and 26 in type $B$.

Lemma 39. We have the following commutation rules for $s_{i} \cdot \Sigma(j)$ for all $1 \leq i \leq n$ and $1 \leq j \leq 2 n$ :

$$
s_{i} \cdot \Sigma(j)= \begin{cases}\Sigma(j) \cdot s_{i} & 1 \leq j<i \text { or } 2 n-i+1<j \leq 2 n \\ \Sigma(j+1) & j=i \text { or } j=2 n-i \\ \Sigma(j-1) & j=i+1 \text { or } j=2 n-i+1 \\ \Sigma(j) \cdot s_{i+1} & i+1<j<2 n-i .\end{cases}
$$

Lemma 40. We have the following product rules for segments in type $C_{n}$ :

1. For $1 \leq j \leq n$,

$$
\Sigma(j) \cdot \Sigma(j)=\Sigma(j-1) \cdot \Sigma(j) \cdot s_{1}
$$


2. For $n+1 \leq j \leq 2 n$,

$$
\Sigma(j+1) \cdot \Sigma(j)=\Sigma(j) \cdot \Sigma(j) \cdot s_{1} .
$$

Theorem 41. In type $C_{n}, \pi: \widetilde{W}^{S} \longrightarrow \mathcal{P}=\mathcal{P}\left(C_{n}\right)$ is a length preserving bijection.

Proof. As in the proof of Theorem 28, we only need to show that the allowed pairs in type $C_{n}$ have parts bounded by $2 n$ and are strictly increasing if the larger part has length less than or equal to $n$. This follows directly from Lemma 40.

Corollary 42. The product $\Sigma(j) \cdot \Sigma(k) \in \widetilde{W}^{S}$ and $\ell(\Sigma(j) \cdot \Sigma(k))=j+k$ if and only if $1 \leq j<k \leq 2 n$ or $n<j=k \leq 2 n$.

Example 43. If $n=4$, the partition $(7,5,5,3,1) \in \mathcal{P}\left(C_{n}\right)$ corresponds with $s_{0} \cdot s_{2} s_{1} s_{0}$. $s_{4} s_{3} s_{2} s_{1} s_{0} \cdot s_{4} s_{3} s_{2} s_{1} s_{0} \cdot s_{2} s_{3} s_{4} s_{3} s_{2} s_{1} s_{0}$. Pictorially, we have

\begin{tabular}{|c|c|c|c|c|}
\hline 0 & 12 & 3 & 4 & \begin{tabular}{|l|l|}
3 & 2 \\
\end{tabular} \\
\hline 0 & 12 & 13 & 4 & \\
\hline 0 & 12 & 13 & 5 & \\
\hline 0 & 12 & & & \\
\hline 0 & & & & \\
\hline
\end{tabular}

where again the corresponding reduced expression is read along the rows from right to left, bottom to top. The columns contain their column number minus one up to column $n+1$ and for $n+1 \leq j \leq 2 n-1$ column $j$ contains $2 n-j+1$.

Bruhat order on $\widetilde{W}^{S}$ in type $C$ is very similar to type $B$. The proofs for the next two lemmas are very similar to the proofs of Lemmas 33 and 34 .

Lemma 44. If $\lambda \in \mathcal{P}\left(C_{n}\right)$ and every $\lambda_{k} \leq n$, then the interval in Bruhat order between id and $\pi^{-1}(\lambda)$ is isomorphic as posets to the interval from the empty partition to $\lambda$ in Young's lattice on strict partitions.

Lemma 45. If $\lambda \in \mathcal{P}\left(C_{n}\right)$ is a single row, then the interval in Bruhat order between id and $\pi^{-1}(\lambda)$ is isomorphic as posets to the interval from the empty partition to $\lambda$ in Young's lattice. In particular, this interval is a totally ordered chain.

Consider the elements in $\widetilde{W}^{S}$ determined by partitions in $\mathcal{P}\left(C_{n}\right)$ for $n \geq 2$. By Lemma 44, the smallest 5 ranks of Bruhat order on $\widetilde{W}^{S}$ is the same as Young's lattice on strict partitions of size at most 4

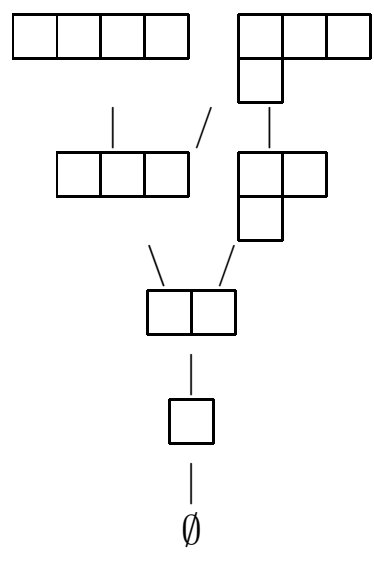


Theorem 46. Let $W$ be the Weyl group of type $C_{n}$ for $n \geq 2$ and let $w \in \widetilde{W}^{S}$. Then $w$ is palindromic if and only if $\pi(w)$ is a one row shape $(j)$ for $0 \leq j \leq 2 n$ or a staircase shape $(k, k-1, k-2, \ldots, 1)$ for $1<k \leq n$.

Remark 47. The number of palindromic elements in $\widetilde{W}^{S}$ for type $C_{n}$ is therefore $3 n$ for $n \geq 2$. The Schubert varieties indexed by one row shapes with $2 \leq j \leq 2 n$ are rationally smooth but not actually smooth. All of the staircase shapes are closed parabolic orbits, hence they are smooth by Theorem 1.

Proof. The proof of Theorem 46 is similar to the proof of Theorem 35. By Lemma 45, we know that $P_{w}(t)=1+t+\cdots+t^{j}$ if $\pi(w)$ is a one row shape, which is clearly palindromic. By Lemma 44 , if $\pi(w)$ is a staircase shape $(k, k-1, k-2, \ldots, 1)$ for $1<k \leq n$ then

$$
P_{w}(t)=\prod_{j=1}^{k}\left(1+t^{j}\right)
$$

which is palindromic.

Conversely, assume $\lambda=\pi(w)$ has at least two rows and is not a staircase shape for $k \leq n$. From (20), we know $a_{1}=a_{2}=1$. If $\lambda$ has two or more $\mathcal{P}$-removable outside corners, then $a_{\ell(w)-1} \geq 2$ so $w$ is not palindromic. Similarly, if $\lambda$ has one $\mathcal{P}$-removable corner and removing it leaves a shape with two $\mathcal{P}$-removable corners, then $a_{\ell(w)-2} \geq 1$ so $w$ is not palindromic. One of these two cases occurs for all partitions $\lambda \in \mathcal{P}\left(C_{n}\right)$ with at least two rows and $\lambda_{1}>n+1$ or if there exists an $i$ such that $\lambda_{i}-\lambda_{i+1} \geq 2$. Therefore, it remains to show that if $\lambda=(n+1)^{k}$ for $k \geq 2$ or $\lambda=\left((n+1)^{k}, n, n-1, \ldots, 1\right)$ for some $k \geq 1$ then $P_{w}(t)$ is not palindromic. Note, these are the only two families of thin elements which are not palindromic in type $C$.

If $\lambda=\left((n+1)^{k}\right)$ for $k \leq n$ then $\lambda$ covers only one element $\mu=\left((n+1)^{k-1}, n\right)$ in Bruhat order. For $k<n, \mu$ covers both $\left((n+1)^{k}, n-1\right)$ and $\left((n+2)^{k-1}, n-k\right)$. The later covering relations is seen by deleting the $s_{n-k}$ from the $\Sigma(n)$ factor which allows the $s_{n-k+1}, \ldots, s_{n-1}$ in the bottom row to commute up adding one generator to each other factor. For $k=n, \mu$ covers $\left((n+2)^{n-1}\right)$ by deleting the $s_{0}$ in the bottom row. Therefore, for all $k \geq 2$ there are at least two elements of rank $|\lambda|-2$ below $\lambda=\left((n+1)^{k}\right)$ in Bruhat order.

Assume now $\lambda=\left((n+1)^{k}, n, n-1, \ldots, 1\right)$. If $k \geq n$ then it follows from the previous case that $\lambda$ is not palindromic. If $0<k<n$, then $\lambda$ covers two elements $\left((n+1)^{k}, n, n-\right.$ $1, \ldots, 2)$ and $\left((n+2)^{k-1}, n, n-1, \ldots, k+2, k, k-1, \ldots 1\right)$. The later element is obtained from $\lambda$ by knocking out the $s_{0}$ in the $\Sigma(k+1)$ factor and allowing the $s_{1}, \ldots, s_{k}$ generators to commute up adding one generator to each of the $\Sigma(n+1)$ factors. Hence, for all $k \geq 1$, $\lambda$ is not palindromic.

Corollary 48. For $W$ of type $C_{n}$, we have $m_{W}=2$. 


\section{Type $D$}

Let $\mathcal{P}\left(D_{n}\right)$ be the set of partitions whose parts are bounded by $2 n-2$, the parts of length at most $n-2$ are strictly decreasing, and the parts of length $n-1$ are two colored, but at most one color can appear in any partition in $\mathcal{P}\left(D_{n}\right)$. The generating function for such partitions is

$$
G_{D_{n}}(x)=\frac{(1+x)\left(1+x^{2}\right) \cdots\left(1+x^{n-2}\right)\left(1+x^{n-1}\right)}{\left(1-x^{n-1}\right)\left(1-x^{n}\right)\left(1-x^{n+1}\right) \cdots\left(1-x^{2 n-2}\right)}
$$

Lemma 49. We have the following generating function identity with Bott's formula from (1):

$$
G_{D_{n}}(x)=\frac{1}{\left(1-x^{n-1}\right)(1-x)\left(1-x^{3}\right)\left(1-x^{5}\right) \cdots\left(1-x^{2 n-3}\right)} .
$$

Proof. The formula holds for $n=4$ and the general case follows by induction.

The Coxeter graph for $\widetilde{D}_{n}$ has an involution interchanging $s_{0}$ and $s_{1}$ so this is a Type II Coxeter graph. The segments described in Section 3 for type $D$ are given as follows: for $1 \leq j \leq 2 n-2, z \in\{b, c\}=\{$ blue, crimson $\}$ set

$$
\Sigma_{1}^{z}(j)= \begin{cases}s_{j} \ldots s_{3} s_{2} s_{1} & 1 \leq j \leq n-2 \\ s_{n-1} s_{n-2} \ldots s_{3} s_{2} s_{1} & j=n-1 \text { and } z=b \\ s_{n} s_{n-2} \ldots s_{3} s_{2} s_{1} & j=n-1 \text { and } z=c \\ s_{2 n-j-1} \cdots s_{n-2} s_{n-1} s_{n} s_{n-2} \cdots s_{4} s_{3} s_{2} s_{1} & n \leq j \leq 2 n-2 .\end{cases}
$$

Note, there are two distinct 1-segments of length $n-1$, namely $\Sigma_{1}^{b}(n-1)$ and $\Sigma_{1}^{c}(n-1)$. The superscript $z=b, c$ distinguishes these two cases. Otherwise, for $j \neq n-1$ the $z$ is irrelevant and can be omitted if it is convenient.

Similarly, define $\Sigma_{0}^{z}(j)$ for all $1 \leq j \leq 2 n-2$ and $z \in\{b, c\}$ to be the reduced expression obtained from $\Sigma_{1}^{z}(j)$ by interchanging $s_{0} \leftrightarrow s_{1}$ and $s_{n-1} \leftrightarrow s_{n}$. For example, $\Sigma_{1}^{b}(n-1)=s_{n-1} s_{n-2} \ldots s_{3} s_{2} s_{1}$ so $\Sigma_{0}^{b}(n-1)=s_{n} s_{n-2} \ldots s_{3} s_{2} s_{0}$. Note the longest 0 segment $\Sigma_{0}^{z}(2 n-2)=s_{0} s_{2} \cdots s_{n-2} s_{n-1} s_{n} s_{n-2} \cdots s_{4} s_{3} s_{2} s_{0}$ both starts and ends in $s_{0}$.

Lemma 50. In type $\widetilde{D}_{n}$, we have the following commutation rules for $s_{i} \cdot \Sigma_{1}^{z}(j)$ for all $1 \leq j \leq 2 n-2$ and $z \in\{b, c\}$. For $1 \leq i \leq n-2$,

$$
s_{i} \cdot \Sigma_{1}^{z}(j)= \begin{cases}\Sigma_{1}^{z}(j) \cdot s_{i} & 1 \leq j<i-1 \text { or } 2 n-i-1<j \leq 2 n-2 \\ \Sigma_{1}^{z}(j+1) & j=i-1 \text { or } j=2 n-i-2 \\ \Sigma_{1}^{z}(j-1) & j=i \text { or } j=2 n-i-1 \\ \Sigma_{1}^{z}(j) \cdot s_{i+1} & i<j<2 n-i-2 .\end{cases}
$$


For $i=n-1$, we have

$$
s_{n-1} \cdot \Sigma_{1}^{z}(j)= \begin{cases}\Sigma_{1}(j) \cdot s_{n-1} & 1 \leq j<n-2 \\ \Sigma_{1}^{b}(j+1) & j=n-2 \\ \Sigma_{1}(j+1) & j=n-1 \text { and } z=c \\ \Sigma_{1}(j-1) & j=n-1 \text { and } z=b \\ \Sigma_{1}^{c}(j-1) & j=n \\ \Sigma_{1}(j) \cdot s_{n} & n<j \leq 2 n-2 .\end{cases}
$$

For $i=n$, the commutation relations for $s_{n} \cdot \Sigma_{1}^{z}(j)$ are similar to those in (23) interchanging $b \leftrightarrow c$ and $s_{n} \leftrightarrow s_{n-1}$. In particular, for $n<j \leq 2 n-2$,

$$
s_{n} \cdot \Sigma_{1}^{z}(j)=\Sigma_{1}^{z}(j) \cdot s_{n-1} .
$$

For $i=0$

$$
\Sigma_{0}^{z}(1) \cdot \Sigma_{1}^{z}(1)=s_{0} s_{1}=s_{1} s_{0}=\Sigma_{1}^{z}(1) \cdot \Sigma_{0}^{z}(1) .
$$

Similarly, commutation rules for $s_{i} \cdot \Sigma_{0}^{z}(j)$ for all $0 \leq i \leq n, z \in\{b, c\}$ and $1 \leq j \leq 2 n-2$ are obtained from (22) and (23) by interchanging $s_{0} \leftrightarrow s_{1}$ and $s_{n-1} \leftrightarrow s_{n}$.

Proof. These follow directly from the commutation relations among the generators determined by the Dynkin diagram.

Lemma 51. We have the following product rules for segments in type $\widetilde{D}_{n}$ :

1. For $1 \leq j<n-1$, we have

$$
\Sigma_{1}(j) \cdot \Sigma_{0}(j)=\Sigma_{1}(j-1) \Sigma_{0}(j) \cdot s_{1} .
$$

2. For $j=n-1$, we have

$$
\Sigma_{1}^{b}(n-1) \cdot \Sigma_{0}^{c}(n-1)=\Sigma_{1}(n-2) \Sigma_{0}^{c}(n-1) \cdot s_{1}
$$

and

$$
\Sigma_{1}^{c}(n-1) \cdot \Sigma_{0}^{b}(n-1)=\Sigma_{1}(n-2) \Sigma_{0}^{b}(n-1) \cdot s_{1} .
$$

3. For $n \leq j<2 n-1$, we have

$$
\Sigma_{1}(j+1) \cdot \Sigma_{0}(j)=\Sigma_{1}(j) \Sigma_{0}(j) \cdot s_{1}
$$

Similar rules hold interchanging $0 \leftrightarrow 1$ above.

Proof. If $1 \leq j<n-1$, then the segments $\Sigma_{0}(j)$ and $\Sigma_{1}(j)$ involve only a proper subset of the generators of $\widetilde{S}$. This proper subset generates a parabolic subgroup isomorphic to a parabolic subgroup of the affine Weyl group of type $\widetilde{B}_{n}$. Therefore, the stated relation holds by Lemma 26. 
Assume $n \leq j<2 n-1$ and let $t=2 n-j-1$. Then,

$$
\begin{aligned}
\Sigma_{0}(j) & =s_{t} s_{t+1} \cdots s_{n-2} s_{n-1} s_{n} s_{n-2} \cdots s_{2} s_{0} \\
\Sigma_{1}(j+1) & =s_{t-1} s_{t} s_{t+1} \cdots s_{n-2} s_{n-1} s_{n} s_{n-2} \cdots s_{2} s_{1} .
\end{aligned}
$$

By Lemma 50, we have the commutation moves $\Sigma_{1}(j+1) s_{i}=s_{i} \Sigma_{1}(j+1)$ for all $t \leq i \leq n-$ $2, \Sigma_{1}(j+1) s_{n-1}=s_{n} \Sigma_{1}(j+1), \Sigma_{1}(j+1) s_{n}=s_{n-1} \Sigma_{1}(j+1)$. Note, $\Sigma_{1}(j+1) s_{t-1}$ is not equal to a single generator times a segment, but $\Sigma_{1}(j+1) s_{t-1}=s_{t-1} \cdot \Sigma_{1}(j) s_{t-1}=s_{t-1} s_{t-2} \cdot \Sigma_{1}(j)$. Then by Lemma 50 again we have $\Sigma_{1}(j) s_{i}=s_{i-1} \Sigma_{1}(j)$ for all $2 \leq i<t-1$. Therefore,

$$
\begin{aligned}
\Sigma_{1}(j+ & 1) \Sigma_{0}(j)=\Sigma_{1}(j+1) \cdot s_{t} s_{t+1} \cdots s_{n-2} s_{n-1} s_{n} s_{n-2} \cdots s_{2} s_{0} \\
& =s_{t} s_{t+1} \cdots s_{n-2} s_{n-1} s_{n} s_{n-2} \cdots s_{t} \cdot \Sigma_{1}(j+1) \cdot s_{t-1} \cdots s_{2} s_{0} \\
& =s_{t} s_{t+1} \cdots s_{n-2} s_{n-1} s_{n} s_{n-2} \cdots s_{t} \cdot s_{t-1} \Sigma_{1}(j) \cdot s_{t-1} \cdots s_{2} s_{0} \\
& =s_{t} s_{t+1} \cdots s_{n-2} s_{n-1} s_{n} s_{n-2} \cdots s_{t} \cdot s_{t-1} \cdot s_{t-2} \cdots s_{1} \cdot \Sigma_{1}(j) \cdot s_{0} \\
& =\Sigma_{1}(j) \cdot \Sigma_{1}(j) \cdot s_{0} \\
& =\Sigma_{1}(j) \cdot s_{t} s_{t+1} \cdots s_{n-2} s_{n-1} s_{n} s_{n-2} s_{n} \cdots s_{2} s_{1} \cdot s_{0} \\
& =\Sigma_{1}(j) \cdot s_{t} s_{t+1} \cdots s_{n} \cdots s_{2} s_{0} \cdot s_{1} \\
& =\Sigma_{1}(j) \cdot \Sigma_{0}(j) \cdot s_{1}
\end{aligned}
$$

since $s_{0}$ and $s_{1}$ commute.

\begin{tabular}{|c|c|c|c|}
\hline 0 & & & 6 \\
\hline 1 & 25 & & 5 \\
\hline 0 & 25 & & 6 \\
\hline 1 & 27 & & \\
\hline
\end{tabular}

For $j=n-1$, the proof is similar to the calculations above.

Theorem 52. In type $D_{n}, \pi: \widetilde{W}^{S} \longrightarrow \mathcal{P}=\mathcal{P}\left(D_{n}\right)$ is a length preserving bijection.

Proof. As in the proof of Theorem 28, we only need to show that the allowed pairs in type $D_{n}$ are colored partitions in $\mathcal{P}\left(D_{n}\right)$ given Lemma 49. This follows directly from Lemma 51.

Corollary 53. The product $\Sigma_{1}^{y}(j) \cdot \Sigma_{0}^{z}(k) \in \widetilde{W}^{S}$ and $\ell\left(\Sigma_{1}(j) \cdot \Sigma_{0}(k)\right)=j+k$ if and only if $y=z$ and $(1 \leq j<k \leq 2 n-2$ or $n-1 \leq j=k \leq 2 n-2)$.

Example 54. If $n=6$, the colored partition $\left(7,5^{b}, 5^{b}, 3,1\right) \in \mathcal{P}\left(D_{n}\right)$ corresponds with $s_{0} \cdot s_{3} s_{2} s_{1} \cdot s_{6} s_{4} s_{3} s_{2} s_{0} \cdot s_{5} s_{4} s_{3} s_{2} s_{1} \cdot s_{4} s_{6} s_{5} s_{4} s_{3} s_{2} s_{0}$. Pictorially, we have

where again the corresponding reduced expression is read along the rows from right to left, bottom to top. Note, the 0's and 1's alternate in the first column starting with a 0 in the top left corner. The columns indexed by $1<j<n-1$ contain $j$, for $n<j<2 n-2$ column $j$ contains $2 n-j-1$, and column $2 n-2$ again alternates from 0 to 1 starting from the top. Columns $n-1$ and $n$ alternate between the values $n-1, n$ as well depending on the unique color of the parts of size $(n-1)$ if they are present.

Bruhat order on $\widetilde{W^{S}}$ in type $D$ is similar to type $B$ up to rank $n-1$. The proofs for the next two lemmas are very similar to the proofs of Lemmas 33 and 34 . 
Lemma 55. If $\lambda \in \mathcal{P}\left(D_{n}\right)$ and every $\lambda_{k} \leq n-1$, then the interval in Bruhat order between id and $\pi^{-1}(\lambda)$ in $\widetilde{W}^{S}$ is isomorphic as posets to the interval from the empty partition to $\lambda$ in Young's lattice on strict partitions.

Lemma 56. If $\lambda \in \mathcal{P}\left(D_{n}\right)$ is a single row, then the interval in Bruhat order between id and $\pi^{-1}(\lambda)$ is isomorphic as posets to the interval from the empty partition to $\lambda$ in the generalized Young's lattice on colored partitions where the parts of size $n-1$ have two possible colors both covering $(n-2)$ and covered by $(n)$. In particular,

$$
P_{w}(t)=\sum_{v \leq w} t^{\ell(w)}=1+t+t^{2}+\cdots+t^{n-2}+2 t^{n-1}+t^{n}+\cdots+t^{2 n-2} .
$$

Consider the elements in $\widetilde{W}^{S}$ determined by their corresponding partitions in $\mathcal{P}\left(D_{n}\right)$ for $n \geq 5$. By Lemma 55, Bruhat order up to rank 3 on $\widetilde{W}^{S}$ is the same as Young's lattice on strict partitions of size at most 3 as in (16). However, for $n=4$, there are 3 colored partitions of size 3 in $\mathcal{P}\left(D_{4}\right)$.

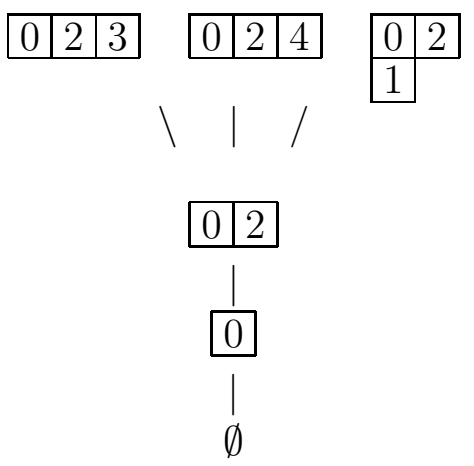

Theorem 57. Let $\widetilde{W}$ be the affine Weyl group of type $D_{n}$ for $n \geq 4$ and let $w \in \widetilde{W}^{S}$. Then $w$ is palindromic if and only if $\pi(w)$ is a one row shape $(j)$ with $1 \leq j \leq n-1$ or $j=2 n-2$ or a staircase shape $(k, k-1, k-2, \ldots, 1)$ for $k \leq n-1$. Note, if $j=n-1$ or $k=n-1$, there are two distinct elements of either shape corresponding to the two possible colors of parts of size $n-1$.

This proof is very similar to Theorem 35. In fact it is a bit easier in this case; to show $P_{w}(t)=\sum a_{i} t^{i}$ is not palindromic it will suffice to show that $a_{\ell(w)-j}>1=a_{j}$ for $j=1$ or $j=2$.

Proof. By Lemma 56, we know that $P_{w}(t)=1+t+\cdots+t^{j}$ if $\pi(w)$ is a one row shape with $0 \leq j \leq n-1$, which is clearly palindromic. Also by Lemma 56 , if $\pi(w)=(2 n-2)$, then $P_{w}(t)$ is palindromic. By Lemma 55 , if $\pi(w)$ is a staircase shape $(k, k-1, k-2, \ldots, 1)$ for $1<k \leq n-1$ then

$$
P_{w}(t)=\prod_{j=1}^{k}\left(1+t^{j}\right)
$$

which is palindromic. 
Conversely, it follows from Lemma 56 that if $\pi(w)$ is a one row shape of length $n \leq j<$ $2 n-2$, then $P_{w}(t)$ is not palindromic. So, assume $\lambda=\pi(w)$ has at least two rows and is not a staircase shape for $k \leq n-1$. From (24), we know $a_{1}=a_{2}=1$. If $\lambda$ has two or more $\mathcal{P}$-removable outside corners, then $a_{\ell(w)-1} \geq 2$ so $w$ is not palindromic. Similarly, if $\lambda$ has one $\mathcal{P}$-removable corner and removing it leaves a shape with two $\mathcal{P}$-removable corners, then $a_{\ell(w)-2} \geq 2$ so $w$ is not palindromic. One of these two cases occurs for all partitions $\lambda \in \mathcal{P}\left(D_{n}\right)$ with at least two rows and $\lambda_{1} \geq n$ or if there exists an $i$ such that $\lambda_{i}-\lambda_{i+1} \geq 2$. Therefore, it remains to show that if $\lambda=\left((n-1)^{k}\right)$ or $\lambda=\left((n-1)^{k},(n-2), \ldots, 1\right)$ for some $k \geq 2$ and with either color pattern, then $P_{w}(t)$ is not palindromic. We can fix the color pattern for the parts of size $n-1$ and look only at partitions with the exact same color pattern in the proof below. Therefore, we will not need to specify the fixed color pattern.

Say $\lambda=\left((n-1)^{k}\right)$ for $2 \leq k \leq n-1$. Then $w$ covers the two elements $\pi^{-1}((n-$ $\left.1)^{k-1}, n-2\right)$ and $\pi^{-1}\left(n^{k-1}, n-k-1\right)$ as seen by omitting the $s_{n-k}$ in the last row of $\lambda$ and allowing all the larger generators to commute up. For example, for $n=6$, we omit the 2 on the last row and allow the $s_{6} s_{4} s_{3}$ to commute up via the relations in Lemma 50

\begin{tabular}{|l|l|l|l|l|}
\hline 0 & 2 & 3 & 4 & 5 \\
\hline 1 & 2 & 3 & 4 & 6 \\
\hline 0 & 2 & 3 & 4 & 5 \\
\hline 1 & & 3 & 4 & 6 \\
\hline
\end{tabular}$\quad \cong$\begin{tabular}{|l|l|l|l|l|l|}
\hline 0 & 2 & 3 & 4 & 5 & 6 \\
\hline 1 & 2 & 3 & 4 & 6 & 5 \\
\hline 0 & 2 & 3 & 4 & 5 & 6 \\
\hline 1 & \multicolumn{7}{|c}{} \\
\hline
\end{tabular}

Hence, $a_{1}=1<2 \leq a_{\ell(w)-1}$.

If $\lambda=\left((n-1)^{k}\right)$ with $k \geq n$, then $w$ covers $v=\pi^{-1}\left((n-1)^{k-1}, n-2\right)$, and $v$ covers two elements since $n \geq 4$, namely the ones corresponding to the partitions $\left((n-1)^{k-1}, n-3\right)$ and $\left((n+1)^{n-2},(n-1)^{k-n}\right)$. The ladder element is obtained by omitting the square in column $n-1$ of row $n$ and the square in column 1 of row $n-1$ of $\lambda$. As in the case of the $\left(n^{n}\right)$ square in the proof of Theorem 35, the remaining generators in rows $n$ and $n-1$ commute up to add two additional squares to each of the first $n-2$ rows. Therefore, $a_{\ell(w)-2} \geq 2>a_{2}=1$. For example, when $n=k=4$ we have

\begin{tabular}{|l|l|l|}
\hline 0 & 2 & 4 \\
\hline 1 & 2 & 3 \\
\hline & 2 & 4 \\
\hline 1 & 2 & \\
\hline
\end{tabular}

On the other hand, say $\lambda=\left((n-1)^{k},(n-2), \ldots, 1\right)$ for some $k \geq 2$. If $k \geq n$, then we can delete two generators in $\lambda$ in two ways as in the proof of Theorem 35 for the shape $\left((n-1)^{n}\right)$ and obtain two elements below $w$ of length $\ell(w)-2$. If $k=2$, then there are two elements of length $\ell(w)-2$ below $w$ corresponding to the partitions $\left((n-1)^{2},(n-2), \ldots, 3,1\right)$ and $(n, n-1, n-2, \ldots, 3)$ obtained from $\lambda$ by deleting the last two elements in column 1 and allowing the $s_{2}$ to commute up. If $2<k<n$, then again $w$ covers two elements corresponding to the partitions $\left((n-1)^{k},(n-2), \ldots, 3,2\right)$ and $\left(n^{k-2},(n-1)^{2}, n-2, \ldots, \widehat{k-1}, \ldots, 1\right)$, the ladder element is obtained from $\lambda$ as follows. Delete the largest generator $s_{k-1}$ in row $n$. The corresponding product of segments has 
two consecutive segments of length $k-2$, use the segment relations $\Sigma_{1}(k-2) \Sigma_{0}(k-2)=$ $\Sigma_{1}(k-3) \Sigma_{0}(k-2) \cdot s_{1}$ or the analogous relation switching 0 and 1 . Then the $s_{1}$ commutes up to row 1 and becomes an $s_{n}$ or $s_{n-1}$ extending row 1. After applying the segment relation, now rows $n+1$ and $n+2$ have the same length. Repeating the same procedure adds an extra box to row 2, etc. After applying $k-2$ segment relations, the remaining partition is $\left(n^{k-2},(n-1)^{2}, n-2, \ldots, \widehat{k-1}, \ldots, 1\right)$.

Corollary 58. For $W$ of type $D_{n}$, we have $m_{W}=n-1$.

Proof. The proof above shows that $n-1$ is sufficient. It is necessary for the element $w=\Sigma_{0}(2 n-3) \in \widetilde{W}^{S}$ with $P_{w}(t)=t^{n}+\sum_{i=0}^{2 n-3} t^{i}$.

\section{Exceptional Types}

In this section we give the algorithm we used to prove Theorem 8 for the exceptional types. As an example of the algorithm, we give the full proof in type $G_{2}$. In type $F_{4}$, we give the necessary data to verify the theorem without much discussion in the appendix. For types $E_{6}, E_{7}, E_{8}$ we refer the reader to our Lisp and Maple code available at

$$
\text { http://www.math.washington.edu/ billey }
$$

Every exceptional type has a Type I Coxeter graph. The segments can be obtained using the Mozes numbers game [4,13]. The node-firing game from [2] can be used as well since the segments form an interval in left weak order so one only needs to find the longest element in $\widetilde{W}^{S}$ with exactly one $s_{0}$ in any reduced expression. The allowed pairs can also be identified using the numbers game or the node-firing game. Below is the high level algorithm for verifying Theorem 8:

1. Identify the set of all segments using the node-firing game.

2. Compute all products of pairs $\Sigma^{c}(i) \Sigma^{d}(j)$ to identify allowed pairs: $\Sigma^{c}(i) \Sigma^{d}(j)$ is allowed if and only if

$$
\ell\left(\Sigma^{c}(i) \Sigma^{d}(j)\right)=i+j
$$

and

$$
\Sigma^{c}(i) \Sigma^{d}(j) \in \widetilde{W^{S}} .
$$

Both tests are straightforward using the numbers game.

3. Let $\mathcal{A}$ be the finite partial order on the allowed colored parts in $\mathcal{P}$ given by $i^{c}<j^{d}$ if $\left(i^{c}, j^{d}\right)$ is an allowed pair. Verify that $\mathcal{A}$ is a subposet of left weak order restricted to the segments confirming Lemma 5 in this case. 
4. Construct a representation of the rational generating function $G_{\mathcal{P}}=\sum_{\lambda \in \mathcal{P}} t^{|\lambda|}$ corresponding with the affine partitions. If $\left(j^{d}, j^{d}\right)$ is an allowed pair, we say $j^{d}$ is repeatable. Let

$$
D\left(j^{c}\right)= \begin{cases}\left(1-t^{j}\right) & j^{c} \text { repeatable } \\ 1 & \text { otherwise. }\end{cases}
$$

and if $\lambda=\left(\lambda_{1}^{c_{1}}>\lambda_{2}^{c_{2}}>\cdots>\lambda_{k}^{c_{k}}\right)$ is a strict colored partition, let $D(\lambda)=$ $\prod_{i=1}^{k} D\left(\lambda_{i}^{c_{i}}\right)$. Then

$$
G_{\mathcal{P}}=\sum \frac{t^{|\lambda|}}{D(\lambda)}
$$

where the sum is over all strictly decreasing chains $\lambda=\left(\lambda_{1}^{c_{1}}>\lambda_{2}^{c_{2}}>\cdots>\lambda_{k}^{c_{k}}\right)$ (including the empty chain) in $\mathcal{A}$ and $|\lambda|=\sum \lambda_{i}$. The sum in (25) is finite since there are only a finite number of segments in each type.

5. Verify that the rational generating function $G_{\mathcal{P}}$ is equal to the expression given by Bott's formula.

Remark 59. In practice a reasonably efficient way to compute the rational generating function in (25) is to identify the set of all strict colored partitions with no repeatable parts and then for each subset of the repeatable parts, check if the repeatable parts can appear with the non-repeatable parts. Furthermore, the strictly decreasing chains of nonrepeatable parts should be constructed using a depth first search that only looks down branches corresponding with allowed pairs.

Example in Type $G_{2}$ : The Dynkin diagram of type $\widetilde{G}_{2}$ is a Type I graph. The segments

\begin{tabular}{|c|c|}
\hline segment & length \\
\hline$\Sigma(1)=s_{0}$ & 1 \\
\hline$\Sigma(2)=s_{1} s_{0}$ & 2 \\
\hline$\Sigma(3)=s_{2} s_{1} s_{0}$ & 3 \\
\hline$\Sigma(4)=s_{1} s_{2} s_{1} s_{0}$ & 4 \\
\hline$\Sigma(5)=s_{2} s_{1} s_{2} s_{1} s_{0}$ & 5 \\
\hline$\Sigma(6)=s_{1} s_{2} s_{1} s_{2} s_{1} s_{0}$ & 6 \\
\hline
\end{tabular}
are as follows:

Observe that the segments have distinct lengths. No colors will be necessary for the corresponding partitions.

One can verify that the product of two segments $\Sigma(i) \cdot \Sigma(j)$ is a reduced expression for a minimal length coset representative in $\widetilde{W}^{S}$ if and only if the ordered pair $(i, j)$ appears in the following list of allowed pairs:

$$
\{(1,4),(1,5),(1,6),(2,5),(2,6),(3,5),(3,6),(4,5),(4,6),(5,5),(5,6),(6,6)\}
$$


Let $\mathcal{P}\left(G_{2}\right)$ be the set of partitions allowed by these pairwise rules, i.e. they have an arbitrary number of 5's and 6's followed by one of the partitions from the following set:

$$
\{(1),(2),(3),(4),(4,1)\}
$$

The generating function for such partitions is

$$
\frac{1+t+t^{2}+t^{3}+t^{4}+t^{1} t^{4}}{\left(1-t^{5}\right)\left(1-t^{6}\right)}=\frac{1+t+t^{2}+t^{3}+t^{4}+t^{5}}{\left(1-t^{5}\right)\left(1-t^{6}\right)}=\frac{1}{\left(1-t^{1}\right)\left(1-t^{5}\right)}
$$

Observe that the formula above agrees with Bott's formula for the rank generating function for $\widetilde{W}^{S}$ since the exponents for type $G_{2}$ are $\{1,5\}$. This proves Theorem 8 for type $G_{2}$.

Above it was easy enough to use the description of $\mathcal{P}\left(G_{2}\right)$ to get the generating function in (27) since 5 and 6 can be added to every affine partition to get another affine partition. In types $F_{4}, E_{6,7,8}$ some repeatable parts are not allowed to appear with other parts so we need to be more careful. For example, in type $F_{4}$ the part denoted $11^{1}$ in the appendix cannot appear with all other parts. Therefore, it is instructive to build this generating function using (25) as an example. The following are all strict affine partitions in $\mathcal{P}\left(G_{2}\right)$ and their corresponding denominators:

$$
\begin{array}{ll}
\{(),(1),(2),(3),(4),(1,4)\} & D(\lambda)=1 \\
\{(5)\} \cup\{(j, 5) \mid 1 \leq j \leq 4\} \cup\{(1,4,5)\} & D(\lambda)=\left(1-t^{5}\right) \\
\{6\} \cup\{(j, 6) \mid 1 \leq j \leq 4\} \cup\{(1,4,6)\} & D(\lambda)=\left(1-t^{6}\right) \\
\{(5,6)\} \cup\{(j, 5,6) \mid 1 \leq j \leq 4\} \cup\{(1,4,5,6)\} & D(\lambda)=\left(1-t^{5}\right)\left(1-t^{6}\right)
\end{array}
$$

Putting this information into (25) we have

$$
G_{\mathcal{P}}=\left(1+t+t^{2}+t^{3}+t^{4}+t^{1} t^{4}\right)\left(1+\frac{t^{5}}{\left(1-t^{5}\right)}+\frac{t^{6}}{\left(1-t^{6}\right)}+\frac{t^{5} t^{6}}{\left(1-t^{5}\right)\left(1-t^{6}\right)}\right)
$$

which simplifies to (27) as expected.

\section{Type $A$}

Fix $W$ to be the Weyl group of type $A_{n}$ and $\widetilde{W}$ its affine Weyl group. The elements in $\widetilde{W}^{S}$ are known to be in bijection with $(n+1)$-core partitions and $n$-bounded partitions $[12,23,19]$. In this section, we will describe the affine partitions in type $A$ and prove that these objects are also in bijection with $\widetilde{W}^{S}$. The proof relies on the $(n+1)$-core bijection. We use the segments to identify the palindromic elements as well. Following a suggestion due to Hugh Thomas, we use the $k$-bounded partitions to identify the Poincaré polynomial for all the palindromic, nonsmooth elements in $\widetilde{W}^{S}$ reproving a theorem due to the second author [24].

In the Dynkin diagram for $\widetilde{A}_{n}$, both $s_{1}$ and $s_{n}$ are connected to $s_{0}$ in the Dynkin diagram for $\widetilde{W}$. We will treat $W$ as a Type I Coxeter group with $J=\left\langle s_{2}, \ldots, s_{n-1}\right\rangle$. The 
fragments in this type are the elements of $W^{J}$. As permutations, these fragments have one line notation in $S_{n+1}$ of the form

$$
[a, 1,2, \ldots, \hat{a}, \ldots, \hat{b}, \ldots, n+1, b]=s_{a-1} s_{a-2} \cdots s_{1} s_{b} s_{b+1} \cdots s_{n-1} s_{n}
$$

where $a, b \in\{1, \ldots, n+1\}:=[n+1]$ and $a \neq b$. The segments can be defined by

$$
\Sigma^{c}(k)=s_{c} s_{c-1} \ldots s_{1} s_{n-k+c+2} \ldots s_{n-1} s_{n} s_{0}
$$

for $0 \leq c \leq n, 1 \leq k-c \leq n$, so $1 \leq k \leq 2 n$ and $k$ is the length of $\Sigma^{c}(k)$. There are $n(n+1)$ segments for type $A_{n}$.

To describe the weak order on segments and the allowed pairs, it is convenient to use an alternative notation. For $0 \leq i \leq n$ and $1 \leq j \leq n$, let

$$
C_{i, j}=s_{i} s_{i-1} \ldots s_{1} s_{n-j+2} \ldots s_{n-1} s_{n} s_{0}
$$

so $\ell\left(C_{i, j}\right)=i+j$ and $\Sigma^{c}(k)=C_{c, k-c}$.

Lemma 60. For $1 \leq a \leq n$, we have the following relations:

$$
s_{a} C_{i, j}= \begin{cases}C_{i, j} s_{a+1} & a<i \text { and } a<n-j \\ C_{i, j+1} & a<i \text { and } a=n-j \\ C_{i, j-1} & a<i \text { and } a=n-j+1 \\ C_{i, j} s_{a} & a<i \text { and } a \geq n-j+2 \\ C_{i-1, j} & a=i \\ C_{i+1, j} & a=i+1 \\ C_{i, j} s_{a} & a>i+1 \text { and } a \leq n-j \\ C_{i, j+1} & a>i+1 \text { and } a=n-j+1 \\ C_{i, j-1} & a>i+1 \text { and } a=n-j+2 \\ C_{i, j} s_{a-1} & a>i+1 \text { and } a>n-j+2 .\end{cases}
$$

Proof. These relations follow directly from the Coxeter relations.

As a direct corollary of the relations above, we get the following characterization of the weak order on segments.

Lemma 61. In left weak order,

$$
C_{i, j} \leq C_{k, l} \Longleftrightarrow \begin{cases}i \leq k \text { and } j \leq l & \text { for } i+j \neq n \\ i<k \text { and } j \leq l & \text { for } i+j=n .\end{cases}
$$

Hence, the left weak order on segments is a poset similar to the product of chains $[n+1] \times[n]$, except that it is missing some relations between the middle two ranks namely, $C_{i, j} \nless C_{i, j+1}$ if $i+j=n$.

Thus, the weak order on segments in type $A$ is significantly more complicated than in all other types. In order to characterize the allowed pairs $C_{i, j} C_{k, l}$ and finish the proof of Theorem 8 in type $A$, we introduce the bijection from $\widetilde{W}^{S}$ to core partitions. 


\subsection{Core Partitions, $k$-bounded partitions, and affine partitions}

Let $\lambda$ be a partition thought of as a Ferrers diagram. We say a square $s$ is addable to $\lambda$ if $s$ is adjacent to the southeast boundary of $\lambda$ and adding $s$ to $\lambda$ results in a larger partition shape. Similarly, a square $s$ inside $\lambda$ is removable from $\lambda$ if $s$ is adjacent to the southeast boundary of $\lambda$ and removing $s$ from $\lambda$ results in a smaller partition shape. The $n$-content of the square $(i, j)$ in matrix notation is $(j-i)$ modulo $(n+1)$ taken in the range $[0, n]$. The arm of a square $s$ in $\lambda$ is the set of boxes strictly to the right of $s$. The leg of a square $s$ in $\lambda$ is the set of boxes strictly below $s$. The hook length of square $s=(i, j)$ in $\lambda$, denoted $h(s)$, is the number of squares weakly below $s$ plus the number of squares strictly to the right of $s$, so $h(s)=|\operatorname{arm}(s)|+|\operatorname{leg}(s)|+1$. A central hook of a partition is any hook whose corner square $s$ is along the main diagonal so $s=(a, a)$ for some $a \in \mathbb{N}$. The lowest central hook of a partition is the central hook with $a$ as large as possible.

An $n+1$-core is a partition with no hook length divisible by $n+1$, or equivalently, no hook length exactly $n+1$ [22]. In type $A_{n}$, Misra and Miwa [23] showed that $(n+1)$-core partitions are in bijection with $\widetilde{W}^{S}$. Lascoux showed that Bruhat order on $\widetilde{W}^{S}$ in type $A_{n}$ is completely determined by the partial order of containment on the corresponding $(n+1)$-cores [20]. See also [3].

Let $C^{n+1}$ be the set of all $(n+1)$-cores. The Misra-Miwa bijection from

$$
\mathfrak{c}: \widetilde{W}^{S} \longrightarrow C^{n+1}
$$

is defined recursively as follows. Consider all squares $(i, j) \in \mathbb{N} \times \mathbb{N}$ labeled by their $n$ content. The empty partition, denoted $\emptyset$, represents the identity element in $\widetilde{W}^{S}$. Say $\mathfrak{c}(w)$ is the $(n+1)$-core for $w \in \widetilde{W}^{S}$ and $s_{i} w \in \widetilde{W}^{S}$ with $\ell(w)<\ell\left(s_{i} w\right)$. To obtain the Ferrers diagram for $\mathfrak{c}\left(s_{i} w\right)$ from the Ferrers diagram for $\mathfrak{c}(w)$, add all of the addable squares of $\mathfrak{c}(w)$ with $n$-content $i$ to $\mathfrak{c}(w)$.

For example, if $n=3$, then the 4-core for $s_{2} s_{3} s_{0}$ is a single column with three boxes obtained by first adding the unique box with $n$-content 0 , then 3 , then 2 in such a way as to obtain a partition shape at each step. The $(n+1)$-cores starting with $s_{2} s_{3} s_{0}$ and building up to $s_{0} s_{3} s_{2} s_{1} s_{2} s_{3} s_{0}$ are given by the following shapes (the $n$-content is written inside each box just for convenience):

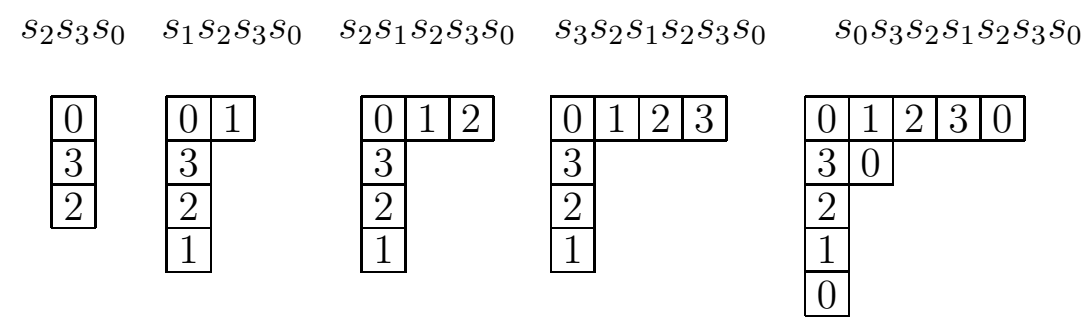

Note, that the number of boxes in $\mathfrak{c}(w)$ is at least $\ell(w)$ but not necessarily equal to $\ell(w)$. More formally, for $0 \leq i \leq n$, let $r_{i}$ be the operator on partitions which acts on $\lambda$ by adding all addable boxes of $n$-content $i$ and removing all removable boxes of $n$-content $i$. If $w \in \widetilde{W^{S}}$ and $w=s_{i_{1}} s_{i_{2}} \ldots s_{i_{p}}$ is a reduced expression, then define $\mathfrak{c}(w)=r_{i_{1}} r_{i_{2}} \ldots r_{i_{p}}(\emptyset)$. 
To see that $\mathfrak{c}: \widetilde{W}^{S} \longrightarrow C^{n+1}$ is a proper map and a bijection we rely on the following facts which are proved in [19].

Theorem 62. Let $w \in \widetilde{W}^{S}$.

1. If $\lambda \in C^{n+1}$ then $r_{i}(\lambda) \in C^{n+1}$ for all $0 \leq i \leq n$ so $\mathfrak{c}(w) \in C^{n+1}$.

2. We have $s_{i} w \in \widetilde{W}^{S}$ if and only if $\mathfrak{c}(w)$ has at least one addable or one removable square of $n$-content $i$. If $w<s_{i} w$, then $\mathfrak{c}(w)$ has no removable squares of $n$-content $i$ and all the addable squares of $n$-content $i$ lie in consecutive diagonals. If $w>s_{i} w$, then $\mathfrak{c}(w)$ has no addable squares of $n$-content $i$ and all the removable squares of $n$-content $i$ lie in consecutive diagonals. If $w \in \widetilde{W}^{S}$ and $s_{i} w \notin \widetilde{W}^{S}$, then $\mathfrak{c}(w)$ has no addable or removable squares with $n$-content $i$.

3. The relations among the set of generators $\widetilde{S}=\left\{s_{0}, s_{1}, \ldots, s_{n}\right\}$ acting on $\widetilde{W^{S}}$ from the left are exactly the same as the relations among the operators $\left\{r_{0}, \ldots, r_{n}\right\}$ acting on $C^{n+1}$. Thus, we can consider the action $s_{i}: C^{n+1} \longrightarrow C^{n+1}$ to be the same as $r_{i}$.

Now we can return to products of segments. Roughly speaking, pairwise allowed products of segments in type $A$ correspond with consecutive central hooks in the core partition.

Lemma 63. The product $C_{i, j} C_{k, l}$ is reduced and in $\widetilde{W}^{S}$ if and only if at least one of the following hold:

1. $i<k$ and $j<l$.

2. $k+l>n$ and $i<k$ and $j \leq l$.

3. $i+j>n$ and $i \leq k$ and $j \leq l$.

Proof. By Theorem 62(2), if $w \in \widetilde{W}^{S}$ and $s_{i} \mathfrak{c}(w)$ adds at least one box, then $s_{i} w \in \widetilde{W^{S}}$, $\ell\left(s_{i} w\right)=\ell(w)+1$, and $\mathfrak{c}(w) \subset s_{i} \mathfrak{c}(w)$. In particular, this shows that every segment $C_{k, l}=s_{k} \cdots s_{1} s_{n-l+2} \cdots s_{n} s_{0}$ has the properties: $C_{k, l} \in \widetilde{W}^{S}, \ell\left(C_{k, l}\right)=k+l$ and $\mathfrak{c}\left(C_{k, l}\right)$ is a single hook shape core partition $\mathfrak{c}\left(C_{k, l}\right)$ with hook length at least $k+l$ and arm length exactly $\mathrm{k}$. The generators $s_{n-l+2} \cdots s_{n} s_{0}$ build up the leg of the hook, and the generators $s_{k} \cdots s_{1}$ build up the arm of the hook. If $k+l>n$ then $\mathfrak{c}\left(C_{k, l}\right)$ has hook length $k+l+1$ since applying the generators $s_{k} \cdots s_{1}$ along the arm of the hook must necessarily also add one more box to the leg of the hook.

Let $H_{0}=\mathfrak{c}\left(C_{k, l}\right), H_{1}=s_{0} \mathfrak{c}\left(C_{k, l}\right), \ldots, H_{i+j}=s_{i} \cdots s_{1} s_{n-j+2} \cdots s_{n} s_{0} \mathfrak{c}\left(C_{k, l}\right)$ be the result of applying each of the generators of $C_{i, j}=s_{i} \cdots s_{1} s_{n-j+2} \cdots s_{n} s_{0}$ from right to left on $\mathfrak{c}\left(C_{k, l}\right)$. Note, $H_{1}$ is a hook plus one extra square on the central diagonal and $H_{i+j}$ is the union of exactly two central hooks since there are no other $s_{0}$ generators in the reduced expression for $C_{i, j}$.

Again by Theorem $62(2)$, the product $C_{i, j} C_{k, l}$ is reduced and in $\widetilde{W}^{S}$ if and only if $H_{a}$ contains at least one square not in $H_{a-1}$ for each $1 \leq a \leq i+j$. Observe, that if 
one of the three conditions above are satisfied, the latter condition holds since the second central hook is built up exactly as it is when applying the generators of $C_{i, j}$ to the empty partition.

Conversely, assume that none of the three conditions hold on $i, j, k, l$. Then there exists a smallest $a$ such that either the lowest squares in both the first and second central hooks of $H_{a-1}$ are in the same row and the next generator tries to add one more square to the leg of the second hook but fails, or the rightmost squares in both the first and second central hooks of $H_{a-1}$ are in the same column and the next generator tries to add one more square to the arm of the second hook but fails. In either case, we claim no new squares are added in $H_{a}$ so $H_{a} \subset H_{a-1}$ hence $C_{i, j} C_{k, l}$ cannot be a reduced factorization for an element in $\widetilde{W^{S}}$.

To prove the claim $H_{a} \subset H_{a-1}$, assume the lowest squares in both the first and second central hooks of $H_{a-1}$ are in the same row and the next generator tries to add one more square in row $r$ and column 2 to the leg of the second hook. The other case is similar. The generator being applied to go from $H_{a-1}$ to $H_{a}$ is $s_{n+1+2-r}$ where $n+1+2-r \geq n-j+2>1$ by definition of the segment $C_{i, j}$. Let $z=n+1+2-r$ so the $n$-content of $(r, 2)$ is $z \bmod (n+1)$. If the cell $(r, 2)$ is not addable then it must be because $(r, 1)$ is not a square in $H_{a-1}$, therefore no square in any row below row $r$ is addable. Square $(2,3)$ has $n$-content 1 so it is not added from $H_{a-1}$ to $H_{a}$ since $z>1$, nor is any square south or east of $(2,3)$. Finally, if a cell of $n$-content $z$ was addable in the first row, then the hook with corner $(1,2)$ would have hook length $n+1$ in $H_{a}$, but this cannot happen by Theorem 62(1). Therefore, $H_{a}$ is contained in $H_{a-1}$.

Rephrasing the vocabulary slightly from Section 3, we say two segments $C_{i, j} C_{k, l}$ form an allowed pair if $\ell\left(C_{i, j} C_{k, l}\right)=i+j+k+l$ and $C_{i, j} C_{k, l} \in \widetilde{W}$. Note, in the language of Section 3, we would have said $\left((i+j)^{i},(k+l)^{k}\right)$ is an allowed pair as a colored partition since $C_{i, j}=\Sigma^{i}(i+j)$. However, in type $A$ it is more convenient to work with the $C_{i, j}$ notation for segments. Furthermore, we say $C_{i_{1}, j_{1}} C_{i_{2}, j_{2}} \cdots C_{i_{p}, j_{p}}$ is an allowed product of segments if each consecutive pair is an allowed pair.

Theorem 64. Let $W$ be the Weyl group of type $A_{n}$. There is a length preserving bijection from $\widetilde{W}^{S}$ to allowed product of segments given by the canonical factorization $r(w)=$ $C_{i_{0}, j_{0}} C_{i_{1}, j_{1}} \cdots C_{i_{p}, j_{p}}$.

Proof. Injectivity of the map $r$ is proved in the first paragraph of the proof for Theorem 8 . Therefore, it remains to show that any allowed product of segments gives rise to a reduced expression for an element in $\widetilde{W}^{S}$.

Let $v=C_{i_{1}, j_{1}} \cdots C_{i_{p}, j_{p}}$ be an allowed product of segments. By induction we can assume $C_{i_{1}, j_{1}} \cdots C_{i_{p}, j_{p}}$ is a reduced factorization for $v$ and $v \in \widetilde{W}^{S}$. Assume $C_{i_{0}, j_{0}} C_{i_{1}, j_{1}}$ is also an allowed product. We will show that $w=C_{i_{0}, j_{0}} v \in \widetilde{W}^{S}$ and $\ell(w)=\ell(v)+i_{0}+j_{0}$.

Construct the $(n+1)$-core partition $\mathfrak{c}(v)$ by multiplying one segment at a time, starting with $C_{i_{p}, j_{p}}$ and ending with $C_{i_{1}, j_{1}}$. By induction, we assume that multiplication by $C_{i_{1}, j_{1}}$ in this construction adds a new square on the main diagonal with $n$-content 0 in square $s=(a, a)$ for some $a$, new squares with $n$-content $n, n-1, \ldots, n-j_{1}+2$, below $s$, and then 
new squares with $n$-content $1,2, \ldots, i_{1}$ to the right of $s$, plus possibly other squares, thus the length of the corresponding element in $\widetilde{W}^{S}$ is strictly increasing with each generator. Similarly, since $C_{i_{0}, j_{0}} C_{i_{1}, j_{1}}$ is also an allowed product, starting with $\mathfrak{c}(v)$ and multiplying by the generators in $C_{i_{0}, j_{0}}$ in reverse order will add at least one new box each with each generator as shown in Lemma 63. The resulting partition will be an $(n+1)$-core $\lambda$. Therefore, the expression $C_{i_{0}, j_{0}} C_{i_{1}, j_{1}} \cdots C_{i_{p}, j_{p}}$ is a reduced factorization for $w=\mathfrak{c}^{-1}(\lambda)$ so $w \in \widetilde{W^{S}}$.

Corollary 65. Assume $w \in \widetilde{W}^{S}$. The factorization $r(w)=C_{i_{1}, j_{1}} C_{i_{2}, j_{2}} \cdots C_{i_{p}, j_{p}}$ can be obtained from $\mathfrak{c}(w)$ by successively removing the lowest central hook of the remaining partition for each factor. To remove a hook, apply generators which remove squares in the lowest central hook along the arm first, then the leg, then the corner square.

Corollary 66. If $r(w)=C_{i_{1}, j_{1}} C_{i_{2}, j_{2}} \cdots C_{i_{p}, j_{p}}$ then $\mathfrak{c}(w)$ has $p$ squares along the main diagonal. Conversely, if $\mathfrak{c}(w)$ has exactly $p$ squares along the main diagonal, then there are $p$ segments in the canonical factorization $r(w)$.

Corollary 67. Let $i: \widetilde{W}^{S} \longrightarrow \widetilde{W}^{S}$ be the involution determined by mapping $s_{i}$ to $s_{n+1-i}$ for $1 \leq i \leq n$. If $w=C_{i_{1}, j_{1}} \cdots C_{i_{p}, j_{p}}$, then $i(w)=C_{j_{1}-1, i_{1}+1} \cdots C_{j_{p}-1, j_{p}+1}$.

Proof. The proof follows directly from the observation that $\mathfrak{c}(w)$ is the transpose of $\mathfrak{c}(i(w))$.

Let $B^{n}$ be the set of $n$-bounded partitions, so $\lambda \in B^{n}$ implies $\lambda_{i} \leq n$ for all $i$. For a fixed $n$, define a map

$$
\mathfrak{b}: C^{n+1} \longrightarrow B^{n}
$$

given by mapping $\lambda \in C^{n+1}$ to $\mu \in B^{n}$ if the Ferrers diagram for $\lambda$ has $\mu_{i}$ squares with hook length at most $n$ in row $i$ for each $i \geq 1$. For example, if $n=3, \lambda=(5,2,1,1,1)$ then $\mathfrak{b}(\lambda)=(3,1,1,1,1)$.

Define a second map,

$$
\mathfrak{a}: B^{n} \longrightarrow \widetilde{W^{S}}
$$

as follows. Given $\mu \in B^{n}$, fill the squares in the Ferrers diagram of $\mu$ by their $n$-content. Say $i_{1} i_{2} \ldots i_{p}$ is the word obtained by reading along the rows of $\mu$ from right to left, bottom to top. Then $\mathfrak{a}(\mu)=s_{i_{1}} s_{i_{2}} \cdots s_{i_{p}}$. For example, with $n=3$,

$$
(3,3)=\begin{array}{l|l|l}
\hline 0 & 1 & 2 \\
\hline 3 & 0 & 1 \\
\hline
\end{array} \quad \Longrightarrow \quad \mathfrak{a}(3,3)=s_{1} s_{0} s_{3} s_{2} s_{1} s_{0}
$$

The next theorem follows from [12, Lemma 23], [19, Theorem 7], and [23].

Theorem 68. The maps $\mathfrak{a}$ and $\mathfrak{b}$ are bijections such that $\mathfrak{a} \circ \mathfrak{b} \circ \mathfrak{c}: \widetilde{W}^{S} \longrightarrow \widetilde{W}^{S}$ is the identity map. Furthermore, if $\mathfrak{a}(\mu)=w \in \widetilde{W}^{S}$ then $|\mu|=\ell(w)$.

Corollary 69. In type $A_{n}$, there exists a size preserving bijection from $n$-bounded partitions to affine partitions $\mathcal{P}$. 
Note, in general the canonical reduced expressions given by the $n$-bounded partition and the corresponding affine partition are different. For example, with $n=3$ again

$$
(2,2,1)=\begin{array}{|l|l}
\hline 0 & 1 \\
\hline 3 & 0 \\
\hline 2 &
\end{array} \quad \Longrightarrow \quad \mathfrak{a}(2,2,1)=s_{2} s_{0} s_{3} s_{1} s_{0}=s_{0} s_{2} s_{1} s_{3} s_{0}=C_{0,1} C_{2,2}
$$

\subsection{Palindromy in type $A$}

Given that the partition bijection $\pi: \widetilde{W}^{S} \longrightarrow \mathcal{P}$ holds in type $A$ by Theorem 8 and Theorem 64 , we can identify all the thin elements of $\widetilde{W}^{S}$ in type $A$ and cpo's relatively easily. Furthermore, we can use the relations in Lemma 60 to identify which of the thin elements are not palindromic. Therefore, we can give a complete characterization of palindromic elements in type $A_{n}$ using the following theorem due to the second author. This allows us to determine $m_{W}$ as well.

Note, that in type $A_{1}, \widetilde{W}^{S}$ is a chain under Bruhat order so every element is thin, extra thin and palindromic. Therefore, throughout the rest of this section we will assume $n \geq 2$.

Theorem 70. [24] In type $A_{n}$ for $n \geq 2$, there exist two infinite families of palindromic elements, namely $\left\{C_{0, j}\left(C_{1, n}\right)^{k}: j+k=0 \bmod n\right\}$ and $\left\{C_{i, 1}\left(C_{n, 1}\right)^{k}: i+k+1=0 \bmod n\right\}$.

We call the palindromic elements in Theorem 70 spiral elements since their unique reduced expressions spiral around the Dynkin diagram clockwise or counterclockwise.

Lemma 71. Assume $W$ has type $A_{n}$ for $n \geq 2$.

1. For each segment $C_{i, j}$ such that $i+j \leq n$ and each $k \geq 0$, the following elements are thin:

$$
\begin{array}{ll}
C_{0, j-i} C_{1, j-i+1} \cdots C_{i-1, j-1} \cdot C_{i, j} \cdot\left(C_{i+1, n-j}\right)^{k} & \text { if } 0 \leq i<j \leq n, \\
C_{0, j-i} C_{1, j-i+1} \cdots C_{i-1, j-1} \cdot C_{i, j} \cdot\left(C_{n+1-i, j}\right)^{k} & \text { if } 0 \leq i<j \leq n, \\
C_{i-j, 1} C_{i-j+1,2} \cdots C_{i-1, j-1} \cdot C_{i, j} \cdot\left(C_{i+1, n-j}\right)^{k} & \text { if } 1 \leq j \leq i<n, \\
C_{i-j, 1} C_{i-j+1,2} \cdots C_{i-1, j-1} \cdot C_{i, j} \cdot\left(C_{n+1-i, j}\right)^{k} & \text { if } 1 \leq j \leq i<n .
\end{array}
$$

2. For each pair $1 \leq i, j \leq n$ such that $i+j=n+1$ and $k \geq 0$, the elements $\left(C_{i, j}\right)^{k}$ are thin.

Conversely, every thin element in $\widetilde{W}^{S}$ is listed above.

Proof. Observe that the branching number $b_{W}=2$, so an affine partition $\lambda=\pi(w) \in \mathcal{P}$ is thin if and only if $\lambda$ covers one element in the generalized Young's lattice.

The only thin segments are of the form $C_{i, j}$ for $i+j=n+1, C_{0, j}$ for $1 \leq j \leq n$, and $C_{i, 1}$ for $0 \leq i<n$. All other segments cover two elements given by reducing the first index or the second index. Furthermore, the segments $C_{i, j}$ such that $i+j>n+1$ all cover two segments $C_{i-1, j}$ and $C_{i, j-1}$ and both are repeatable. So, if $w$ has any segment of length strictly longer than $n+1$ then $w$ is not thin.

Therefore, $w=C_{i_{1}, j_{1}} \cdots C_{i_{p}, j_{p}} \neq \mathrm{id}$ is thin if and only if all of the following hold: 
1. $i_{1}=0$ or $j_{1}=1$ or $i_{1}+j_{1}=n+1$.

2. For each $1 \leq k \leq p$, we have $i_{k}+j_{k} \leq n+1$.

3. For each $1 \leq k<p$, the pair $\left(i_{k+1}, j_{k+1}\right)$ is minimal so that $C_{i_{k}, j_{k}} C_{i_{k+1}, j_{k+1}}$ is an allowed pair.

The lemma now follows from these three conditions.

Lemma 72. For each segment $C_{i, j}$ such that $i+j \leq n$ there exists a cpo with $C_{i, j}$ as its longest segment given by the product

$$
\begin{array}{ll}
C_{0, j-i} C_{1, j-i+1} \cdots C_{i-1, j-1} C_{i, j} & \text { if } i<j \\
C_{i-j, 1} C_{i-j+1,2} \cdots C_{i-1, j-1} C_{i, j} & \text { if } i \geq j .
\end{array}
$$

Furthermore, all cpo's are of this form, hence there are $\frac{n(n+1)}{2}$ of them.

Proof. First note that since $i+j \leq n$, the support of $C_{i, j}$, namely $I_{i, j}=\left\{s \in \widetilde{S}: s \leq C_{i, j}\right\}$, is a proper subset of $\tilde{S}$, and the support of any segment smaller than $C_{i, j}$ in weak order is contained in $I_{i, j}$. Any allowed product of segments with support in $I_{i, j}$ would have length no longer than the products in (28) by Lemma 63. Therefore, by Theorem 64, the product above is the unique cpo with support $I_{i, j}$.

Conversely, given an arbitrary cpo $w$ with support $I \subset \tilde{S}, I \neq \tilde{S}$, let $r(w)=$ $C_{i_{1}, j_{1}} \cdots C_{i_{p}, j_{p}}$ be the corresponding reduced factorization into segments. If $\ell\left(C_{i_{p}, j_{p}}\right)>n$ then its support is all of $\tilde{S}$ by definition of $C_{i_{p}, j_{p}}$, contradiction. Therefore, $i_{p}+j_{p} \leq n$ and $r(w)$ must be one of the products above.

Lemma 73. Assume $W$ has type $A_{n}$ for $n \geq 2$.

1. For each segment $C_{i, j}$ such that $i+j \leq n$ and each $k \geq 1$, the following elements are thin but not palindromic:

$$
\begin{array}{ll}
C_{0, j-i} C_{1, j-i+1} \cdots C_{i-1, j-1} \cdot C_{i, j} \cdot\left(C_{i+1, n-j}\right)^{k} & \text { if } 1 \leq i<j<n, \\
C_{0, j-i} C_{1, j-i+1} \cdots C_{i-1, j-1} \cdot C_{i, j} \cdot\left(C_{n+1-i, j}\right)^{k} & \text { if } 1 \leq i<j<n, \\
C_{i-j, 1} C_{i+1,2} \cdots C_{i-1, j-1} \cdot C_{i, j} \cdot\left(C_{i+1, n-j}\right)^{k} & \text { if } 2 \leq j \leq i<n, \\
C_{i-j, 1} C_{i+1,2} \cdots C_{i-1, j-1} \cdot C_{i, j} \cdot\left(C_{n+1-i, j}\right)^{k} & \text { if } 2 \leq j \leq i<n .
\end{array}
$$

2. For each $0 \leq i<n, 1 \leq j<n$, and $k>0$ such that $i+k+1 \neq 0 \bmod n$ and $j+k \neq 0 \bmod n$, the elements $C_{i, 1}\left(C_{n, 1}\right)^{k}$ and $C_{0, j}\left(C_{1, n}\right)^{k}$ are thin but not palindromic.

3. For each pair $1 \leq i, j \leq n$ such that $i+j=n+1, k \geq 1, k \neq 0 \bmod n$, the elements $\left(C_{i, j}\right)^{k}$ are thin but not palindromic. 
Proof. Using the relations on generators and segments from Lemma 60 and arguments similar to ones used in other types, one can show that every element $w$ in the list above either covers 2 or more elements in the Bruhat order or $w$ covers one element $v$ and $v$ covers 3 elements. Comparing with Bott's formula (1) shows $w$ cannot be palindromic.

For example, consider elements of the form $w=\left(C_{1, n}\right)^{k}$ for all $k \geq 1$ and $k \neq$ $0 \bmod n$. First, assume that $1 \leq k<n$. We claim $w$ covers $v=C_{1, n-k}\left(C_{2, n}\right)^{k-1}$ and $v^{\prime}=C_{0, n}\left(C_{1, n}\right)^{k-1}$. To see the first covering relation, recall $C_{1, n}=s_{1} s_{2} \cdots s_{n} s_{0}$ and knock out the leftmost $s_{k+1}$ generator from the left in $r(w)$. The leftmost $s_{k}$ then commutes to the right decreasing it's index as it passes each $C_{1, n}$ until it becomes an $s_{2}$ which can glue onto the rightmost $C_{1, n}$ forming a $C_{2, n}$. Then the leftmost $s_{k-1}$ commutes right until it becomes an $s_{2}$ etc. Second, for the case $k>n$, we make the following observation from the relations on generators:

$$
C_{2, n}\left(C_{1, n}\right)^{n}=\left(C_{1, n}\right)^{n} C_{2, n}
$$

and

$$
C_{2, n}\left(C_{1, n}\right)^{k} \notin \widetilde{W}^{S} \text { for } 1 \leq k<n .
$$

Therefore, for any $w=\left(C_{1, n}\right)^{k}$ with $k>n$ and $k \neq 0 \bmod n$, we can apply the procedure in the first case to the leftmost $(k \bmod n)$ segments and commute the $C_{2, n}$ 's to the right past any factors of the form $\left(C_{1, n}\right)^{n}$. Hence, $w$ must actually cover at least 2 elements in Bruhat order, so it is not palindromic.

Let $\left[\begin{array}{l}n \\ k\end{array}\right]_{t}$ denote the $t$-analog of $\left(\begin{array}{l}n \\ k\end{array}\right)$

$$
\left[\begin{array}{l}
n \\
k
\end{array}\right]_{t}=\frac{[n] !}{[k] ![n-k] !}
$$

where $[n] !=\prod_{i=0}^{n-1}\left(1+t+t^{2}+\cdots+t^{i}\right)$ is the usual $t$-analog of $n$ !. The polynomial $\left[\begin{array}{c}n \\ k\end{array}\right]_{t}$ gives the rank generating function for the partitions that fit inside a $k \times n-k$ rectangle [28, Sect. 1.3], hence it is palindromic. The polynomial is also the Poincaré polynomial for the Grassmannian manifold $G_{n, k}$.

The following lemma was originally proved in [24]. This short proof was suggested by Hugh Thomas.

Lemma 74. Let $w$ be a spiral element of length $k \cdot n$ for $k \geq 1$. Then

$$
P_{w}(t)=\left[\begin{array}{c}
n+k \\
k
\end{array}\right]_{t} .
$$

Hence $w$ is palindromic.

Proof. Let $w$ be the spiral element $C_{n-k-1,1}\left(C_{n, 1}\right)^{k}$ for some $k \geq 1$. Then $\mathfrak{a}^{-1}(w)=(n)^{k}$. Every partition $\mu \subset(n)^{k}$ has the property that $\mathfrak{a}(\mu) \leq w$ in Bruhat order since the reduced expression corresponding to the $n$-bounded partition $\mu$ is a subexpression of $C_{n-k-1,1}\left(C_{n, 1}\right)^{k}$. Therefore, to prove the lemma, we only need to show that no $\lambda \in B^{n}$ exists such that $\lambda \not \subset(n)^{k}$ and $\mathfrak{a}(\lambda)<w$. 
Given any $n$-bounded partition $\lambda$ such that $\lambda \not \subset(n)^{k}$, then $\lambda$ must have more than $k$ parts. If the canonical reduced expression $\mathfrak{a}(\lambda)=s_{i_{1}} \cdots s_{i_{p}}$, then the core partition $r_{i_{1}} \cdots r_{i_{p}}(\emptyset)=\mathfrak{b}^{-1}(\lambda)$ must have at least $k+1$ rows. However, by similar logic $\mathfrak{b}^{-1}\left((n)^{k}\right)$ has exactly $k$ rows, therefore $\mathfrak{a}(\lambda) \not{Z} \mathfrak{a}\left((n)^{k}\right)=w$ by Lascoux's Theorem mentioned earlier: Bruhat order on $\widetilde{W}^{S}$ in type $A_{n}$ is completely determined by the partial order of containment on the corresponding $(n+1)$-cores [20].

The proof for $w=C_{0, n-k}\left(C_{1, n}\right)^{k}$ follows from the natural involution on the Dynkin diagram fixing $s_{0}$.

Theorem 75. Let $W$ be the Weyl group of type $A_{n}$. Every element in $\widetilde{W}^{S}$ is palindromic if $n=1$. If $n \geq 2$, then the palindromic elements in $\widetilde{W}^{S}$ are precisely the cpo's and the spiral elements.

Proof. Lemma 72 and Lemma 74 prove each of the cpo's and spiral elements are palindromic. Lemmas 71 and 73 show that every other element is not palindromic.

Corollary 76. For $W$ of type $A_{n}$ with $n \geq 2$, we have $m_{W}=2$.

\section{Appendix}

Example in type $F_{4}$ : The segments for $F_{4}$ appear in Equation (30). Observe that the segments do not have distinct lengths, so colors will be necessary for the corresponding partitions.

One can verify that the product of two segments $\Sigma(i) \cdot \Sigma(j)$ is a reduced expression for a minimal length coset representative in $\widetilde{W}^{S}$ if and only if the ordered pair $(i . j)$ appears in the list of allowed patterns in Figure 10.

This list of allowed patterns is harder to internalize than in types $B, C, D, G_{2}$, however some useful information pops out of it. First, the only parts which can repeat in a colored partition are $11^{1}, 14,15$ and 16 . Additional parts indicated by $14,15,16$ can be added to any affine partition to get another affine partition, but $11^{1}$ cannot appear in any partition with $8^{1}, 9,10,11$ or 12 . Let $N R$ be the set of allowed partitions with no parts in the set $\left\{11^{1}, 14,15,16\right\}$. There are 132 partitions in $N R$ and they all have size at most 40 . The colored partition $\left(13,12^{1}, 10^{1}, 5^{1}\right)$ is the unique one in $N R$ with size 40 exactly. Let $N R E$

be the set of all partitions in $N R$ that can be in an allowed partition with $11^{1}$. There are 96 elements in NRE including $\left(13,12^{1}, 10^{1}, 5^{1}\right)$.

To compute the generating function for $\mathcal{P}\left(F_{4}\right)$, let

$$
G_{N R}(t)=\sum_{\lambda \in N R} t^{|\lambda|}
$$

be the rank generating function for the partitions in $N R$ and similarly for $N R E$. Then, since every partition in $\mathcal{P}\left(F_{4}\right)$ contains an $11^{1}$ or not, we get the following identity

$$
G_{\mathcal{P}\left(F_{4}\right)}=\frac{G_{N R}}{\left(1-t^{14}\right)\left(1-t^{15}\right)\left(1-t^{16}\right)}+\frac{t^{11} \cdot G_{N R E}}{\left(1-t^{11}\right)\left(1-t^{14}\right)\left(1-t^{15}\right)\left(1-t^{16}\right)} .
$$


By computer it is easy to compute the coefficients for $G_{N R}$ and $G_{N R E}$

$N R \quad(11111223334455554556666655443222322221111)$

NRE (111112223222223332345544433332122322221111)

Miraculously using Maple, the sum in (29) simplifies to

$$
\frac{1}{\left(1-t^{1}\right)\left(1-t^{5}\right)\left(1-t^{7}\right)\left(1-t^{11}\right)} \text {. }
$$

as expected using Bott's formula since the exponents for $F_{4}$ are $1,5,7,11$. This proves Theorem 8 for type $F_{4}$.

The nine palindromic elements of $F_{4}$ are given by the affine partitions

$$
\text { (), (1), (2), (3), (4), (5), ( } \left.5^{1}\right),(6),(6,1) \text {. }
$$

\begin{tabular}{|c|c|}
\hline segment & length \\
\hline$\Sigma(1)=s_{0}$ & 1 \\
\hline$\Sigma(2)=s_{1} s_{0}$ & 2 \\
\hline$\Sigma(3)=s_{2} s_{1} s_{0}$ & 3 \\
\hline$\Sigma(4)=s_{3} s_{2} s_{1} s_{0}$ & 4 \\
\hline$\Sigma(5)=s_{2} s_{3} s_{2} s_{1} s_{0}$ & 5 \\
\hline$\Sigma^{1}(5)=s_{4} s_{3} s_{2} s_{1} s_{0}$ & 5 \\
\hline$\Sigma(6)=s_{1} s_{2} s_{3} s_{2} s_{1} s_{0}$ & 6 \\
\hline$\Sigma^{1}(6)=s_{2} s_{4} s_{3} s_{2} s_{1} s_{0}$ & 6 \\
\hline$\Sigma(7)=s_{1} s_{2} s_{4} s_{3} s_{2} s_{1} s_{0}$ & 7 \\
\hline$\Sigma^{1}(7)=s_{3} s_{2} s_{4} s_{3} s_{2} s_{1} s_{0}$ & 7 \\
\hline$\Sigma(8)=s_{1} s_{3} s_{2} s_{4} s_{3} s_{2} s_{1} s_{0}$ & 8 \\
\hline$\Sigma^{1}(8)=s_{2} s_{3} s_{2} s_{4} s_{3} s_{2} s_{1} s_{0}$ & 8 \\
\hline$\Sigma(9)=s_{1} s_{2} s_{3} s_{2} s_{4} s_{3} s_{2} s_{1} s_{0}$ & 9 \\
\hline$\Sigma^{1}(9)=s_{2} s_{1} s_{3} s_{2} s_{4} s_{3} s_{2} s_{1} s_{0}$ & 9 \\
\hline$\Sigma(10)=s_{2} s_{1} s_{2} s_{3} s_{2} s_{4} s_{3} s_{2} s_{1} s_{0}$ & 10 \\
\hline$\Sigma^{1}(10)=s_{3} s_{2} s_{1} s_{3} s_{2} s_{4} s_{3} s_{2} s_{1} s_{0}$ & 10 \\
\hline$\Sigma(11)=s_{3} s_{2} s_{1} s_{2} s_{3} s_{2} s_{4} s_{3} s_{2} s_{1} s_{0}$ & 11 \\
\hline$\Sigma^{1}(11)=s_{4} s_{3} s_{2} s_{1} s_{3} s_{2} s_{4} s_{3} s_{2} s_{1} s_{0}$ & 11 \\
\hline$\Sigma(12)=s_{2} s_{3} s_{2} s_{1} s_{2} s_{3} s_{2} s_{4} s_{3} s_{2} s_{1} s_{0}$ & 12 \\
\hline$\Sigma^{1}(12)=s_{4} s_{3} s_{2} s_{1} s_{2} s_{3} s_{2} s_{4} s_{3} s_{2} s_{1} s_{0}$ & 12 \\
\hline$\Sigma(13)=s_{2} s_{4} s_{3} s_{2} s_{1} s_{2} s_{3} s_{2} s_{4} s_{3} s_{2} s_{1} s_{0}$ & 13 \\
\hline$\Sigma(14)=s_{3} s_{2} s_{4} s_{3} s_{2} s_{1} s_{2} s_{3} s_{2} s_{4} s_{3} s_{2} s_{1} s_{0}$ & 14 \\
\hline$\Sigma(15)=s_{2} s_{3} s_{2} s_{4} s_{3} s_{2} s_{1} s_{2} s_{3} s_{2} s_{4} s_{3} s_{2} s_{1} s_{0}$ & 15 \\
\hline$\Sigma(16)=s_{1} s_{2} s_{3} s_{2} s_{4} s_{3} s_{2} s_{1} s_{2} s_{3} s_{2} s_{4} s_{3} s_{2} s_{1} s_{0}$ & 16 \\
\hline
\end{tabular}

Using (30), we see the cpo's correspond with ()$,(1),(2),(3),(6,1)$. 


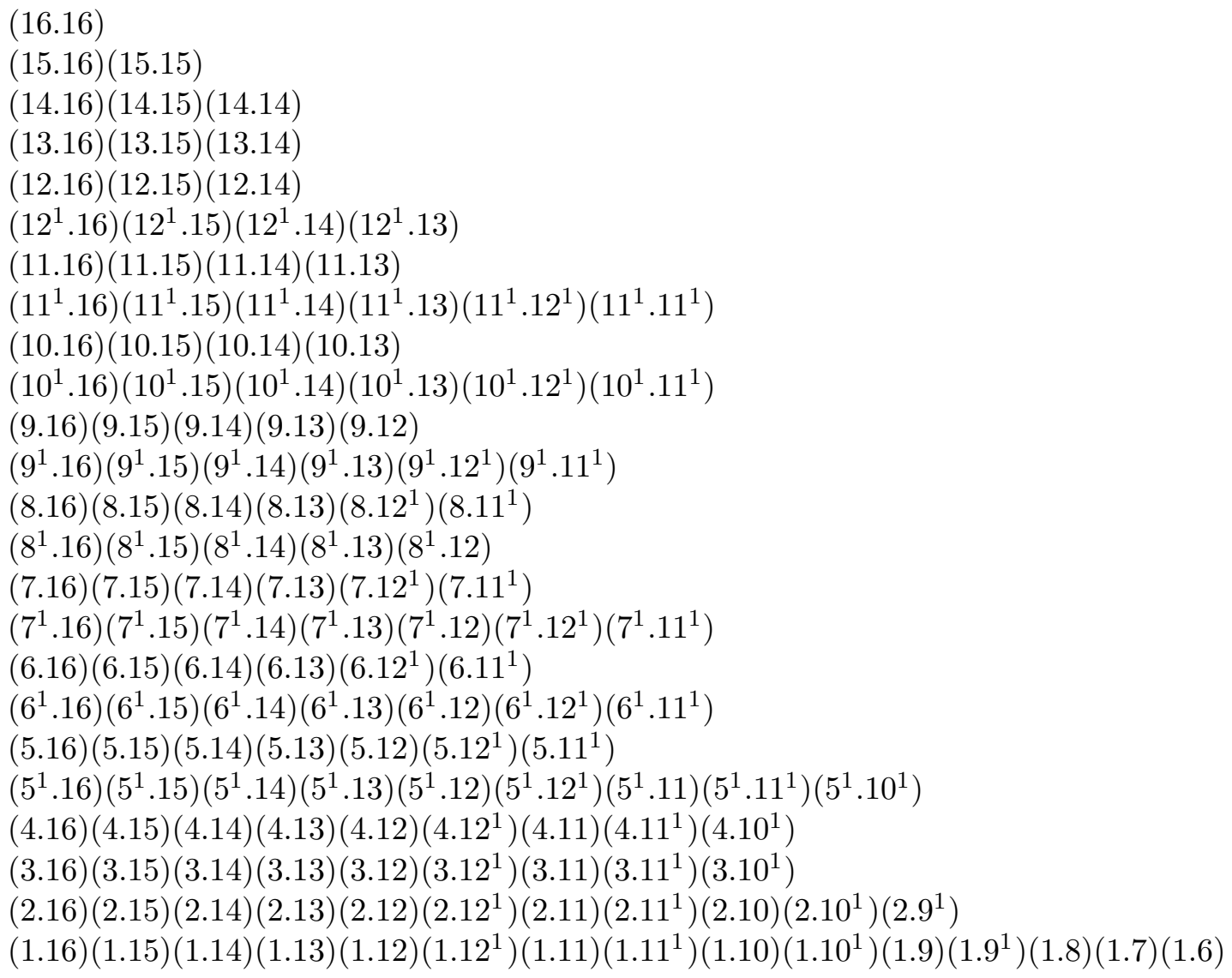

Figure 1: Allowed pairs for $F_{4}$. 
Figure 2: Affine Dynkin diagrams

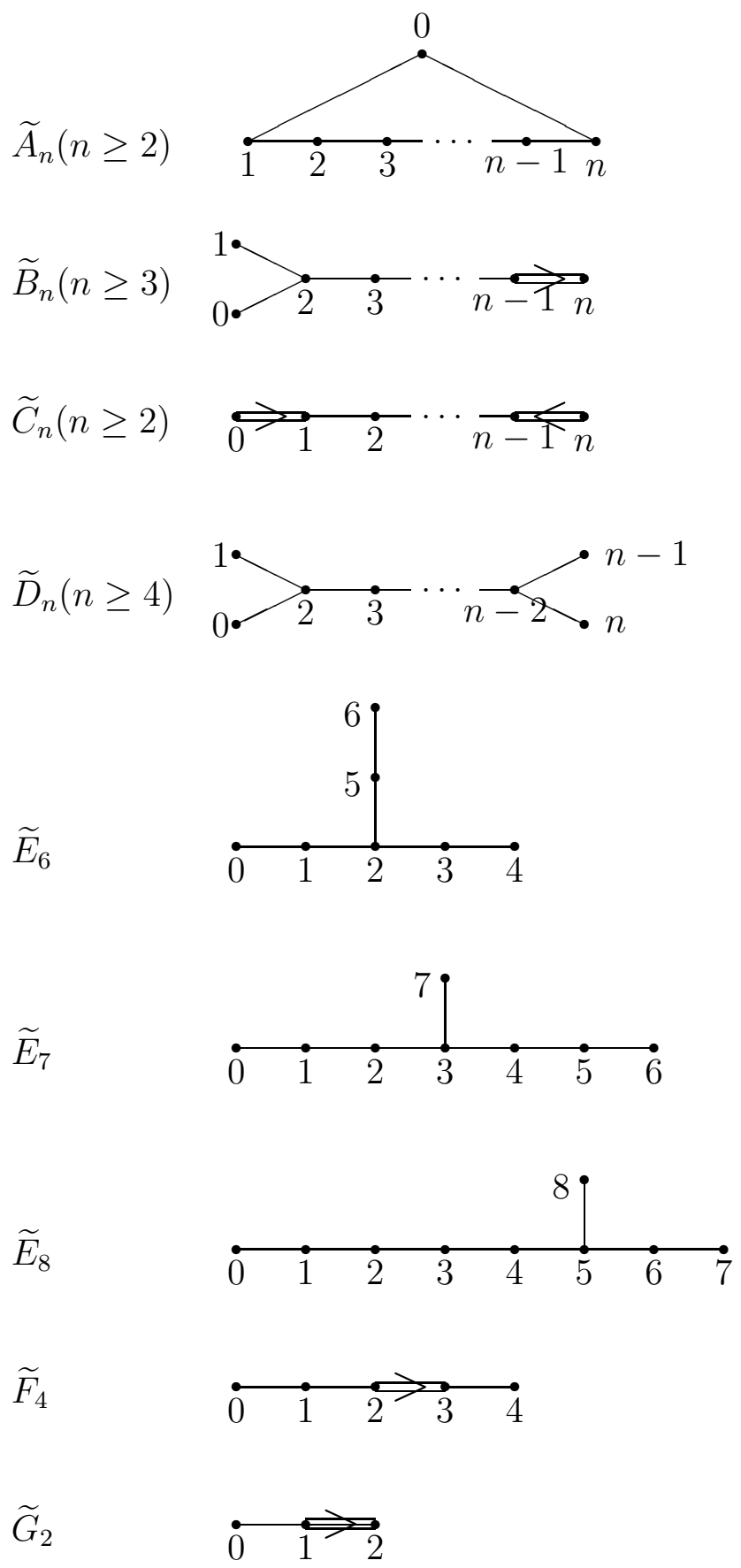




\section{Acknowledgments}

Many thanks to Andrew Crites, Adriano Garsia, Thomas Lam, Mark Shimozono and the anonymous referees for helpful suggestions to this work. Special thanks to Hugh Thomas for the short proof of Lemma 74.

\section{References}

[1] G. E. Andrews, The Theory of Partitions, Cambridge Mathematical Library, Cambridge University Press, Cambridge, 1998. Reprint of the 1976 original.

[2] S. Billey And S. Mitchell, Smooth and palindromic Schubert varieties in affine Grassmannians, To appear in J. Algebraic Combinatorics, (2009).

[3] A. Buörner And F. Brenti, Affine permutations of type A, Electron. J. Combin., 3 (1996), pp. Research Paper 18, approx. 35 pp. (electronic). The Foata Festschrift.

[4] A. Buörner And F. BREnti, Combinatorics of Coxeter groups, vol. 231 of Graduate Texts in Mathematics, Springer, New York, 2005.

[5] A. BuÖrner And T. Ekedahl, On the shape of Bruhat intervals, Annals of Math, (to appear).

[6] R. BotT, An application of the Morse theory to the topology of Lie groups, Bull. Soc. Math. France, 84 (1956), pp. 251-281.

[7] N. Bourbaki, Chapitre IV: Groupes de Coxeter et systmes de Tits. Chapitre V: Groupes engendrs par des rflexions. Chapitre VI: systmes de racines., in Groupes et algbres de Lie, /'El/'ements de math/'ematique, Hermann, Paris, 1968.

[8] M. Bousquet-MÉlou And K. ERIKsson, Lecture hall partitions, Ramanujan J., 1 (1997), pp. 101-111.

[9] —, Lecture hall partitions. II, Ramanujan J., 1 (1997), pp. 165-185.

[10] — A refinement of the lecture hall theorem, J. Combin. Theory Ser. A, 86 (1999), pp. 63-84.

[11] J. B. CARRELL, The Bruhat graph of a Coxeter group, a conjecture of Deodhar, and rational smoothness of Schubert varieties, Proceedings of Symposia in Pure Math., 56 (1994), pp. 53-61.

[12] H. Eriksson And K. ERIKSson, Affine Weyl groups as infinite permutations, Electron. J. Combin., 5 (1998), pp. Research Paper 18, 32 pp. (electronic).

[13] K. ERIKsson, The numbers game and Coxeter groups, Discrete Math., 139 (1995), pp. 155-166. Formal power series and algebraic combinatorics (Montreal, PQ, 1992).

[14] W. Fulton, Young Tableaux; With Applications To Representation Theory And Geometry, vol. 35 of London Mathematical Society Student Texts, Cambridge University Press, New York, 1997.

[15] J. E. Humphreys, Reflection groups and Coxeter groups, Cambridge University Press, 1990. 
[16] S. Kumar, The nil Hecke ring and singularity of Schubert varieties, Inventiones Math., 123 (1996), pp. 471-506.

[17] T. Lam, L. Lapointe, J. Morse, and M. Shimozono, Affine insertion and Pieri rules for the affine Grassmannian, Memoirs of the AMS, (to appear).

[18] T. LAm, A. Schilling, And M. Shimozono, Schubert polynomials for the affine Grassmannian of the symplectic group, Math. Z., (to appear).

[19] L. Lapointe And J. Morse, Tableaux on $k+1$-cores, reduced words for affine permutations, and $k$-Schur expansions, J. Combin. Theory Ser. A, 112 (2005), pp. 4481.

[20] A. LAscoux, Ordering the affine symmetric group, in Algebraic combinatorics and applications (Gößweinstein, 1999), Springer, Berlin, 2001, pp. 219-231.

[21] P. Littig And S. Mitchell, Generating varieties for affine grassmannians, preprint, (arXiv:0810.3266).

[22] I. Macdonald, Symmetric Fuctions and Hall Polynomials, Oxford University Press, Oxford, 1979.

[23] K. Misra And T. Miwa, Crystal base for the basic representation of $U_{q}(\mathfrak{s l}(n))$, Comm. Math. Phys., 134 (1990), pp. 79-88.

[24] S. A. Mitchell, A filtration of the loops on $\mathrm{SU}(n)$ by Schubert varieties, Math. Z., 193 (1986), pp. 347-362.

[25] R. A. Proctor, Classical Bruhat orders and lexicographic shellability, Journal of Algebra, 77 (1982), pp. 104-126.

[26] W. A. PruetT, Canonical reduced expressions and rationally smooth Schubert varieties in affine Grassmannians of simply laced type, manuscript, (June, 2008).

[27] V. REINER, The distribution of descents and length in a Coxeter group, Electron. J. Combin., 2 (1995).

[28] R. P. Stanley, Enumerative Combinatorics. Vol. 1, vol. 49 of Cambridge Studies in Advanced Mathematics, Cambridge University Press, Cambridge, 1997.

[29] - Enumerative Combinatorics. Vol. 2, vol. 62 of Cambridge Studies in Advanced Mathematics, Cambridge University Press, Cambridge, 1999. 